\title{
Emergence of Dirac composite fermions: Dipole picture
}

\author{
Guangyue $\mathrm{Ji} \circledast^{1}$ and Junren $\mathrm{Shi}^{1,2, *}$ \\ ${ }^{1}$ International Center for Quantum Materials, Peking University, Beijing 100871, China \\ ${ }^{2}$ Collaborative Innovation Center of Quantum Matter, Beijing 100871, China
}

(Received 5 July 2021; revised 28 September 2021; accepted 29 September 2021; published 21 October 2021)

\begin{abstract}
Composite fermions (CFs) are the particles underlying the novel phenomena observed in partially filled Landau levels. Both microscopic wave functions and semiclassical dynamics suggest that a CF is a dipole consisting of an electron and a double $2 h / e$ quantum vortex, and its motion is subject to a Berry curvature that is uniformly distributed in the momentum space. Based on the picture, we study the electromagnetic response of composite fermions. We find that the response in the long-wavelength limit has a form identical to that of the Dirac CF theory. To obtain the result, we show that the Berry curvature contributes a half-quantized Hall conductance, which, notably, is independent of the filling factor of a Landau level and not altered by the presence of impurities. The latter is because CFs undergo no side-jumps when scattered by quenched impurities in a Landau-level with the particle-hole symmetry. The remainder of the response is from an effective system that has the same Fermi wave vector, effective density, Berry phase, and therefore long-wavelength response to electromagnetic fields as a Dirac CF system. By interpreting the half-quantized Hall conductance as a contribution from a redefined vacuum, we can explicitly show the emergence of a Dirac CF effective description from the dipole picture. We further determine corrections due to electric quadrupoles and magnetic moments of CFs and show deviations from the Dirac CF theory when moving away from the long-wavelength limit.
\end{abstract}

DOI: 10.1103/PhysRevResearch.3.043055

\section{INTRODUCTION}

The fractional quantum Hall (FQH) effect, a phenomenon discovered nearly four decades ago [1], remains unique as the only topological effect driven by electron correlations and observed in laboratories. In two dimensions, electrons subjected to a strong magnetic field are confined in a Landau level (LL). In such a system, the kinetic energy is completely quenched, and the electron-electron interaction dominates its behavior. Surprisingly, according to the theory of composite fermions [2], the system can be interpreted as a noninteracting or weakly-interacting system consisting of fictitious particles called composite fermions (CFs). It is hypothesized that these particles are bound states of electrons and quantum vortices, reside in a hidden Hilbert space, and form simple quantum states [3]. The theory of CFs prescribes an ansatz for mapping a state in the hidden Hilbert space to a quantum state of a partially filled LL in the real world. The theory is very successful: by interpreting FQH states as integer quantum Hall states of free CFs, wave functions constructed from the ansatz reliably achieve high overlaps with those determined by exact diagonalizations. The theory also prompts experimental searches for CFs. In various geomet-

\footnotetext{
*junrenshi@pku.edu.cn

Published by the American Physical Society under the terms of the Creative Commons Attribution 4.0 International license. Further distribution of this work must maintain attribution to the author(s) and the published article's title, journal citation, and DOI.
}

ric resonance experiments and ballistic transport experiments [4-7], CFs do exhibit behaviors expected for genuine particles. One could treat CFs as if they are elementary particles in the hidden Hilbert space, just like electrons are to the real world.

To treat CFs as the elementary particles of the hidden Hilbert space, one needs an effective theory to specify their dynamics. For a long time, the de facto standard effective theory for CFs is the one proposed by Halperin, Lee, and Read (HLR) [8]. The theory underlies the designs and interpretations of most CF experiments [4,5]. In the HLR theory, CFs feel an effective magnetic field and behave just like ordinary particles by following the ordinary Newtonian dynamics. Such a picture naturally emerges from the Chern-Simons (CS) field theory of CFs, which tries to justify the CF picture by interpreting the electron-vortex binding as a singular CS gauge transformation [9-11]. However, the CS theory disregards the fact that the physics occurs essentially in the projected Hilbert space of a partially filled LL. It contains artifacts, which have to be eliminated in the HLR theory. For instance, to remove the unwelcome presence of the bare band mass of electrons without introducing inconsistencies, one has to assume phenomenologically that a mass renormalization is accompanied by a corresponding renormalization to the effective interaction between CFs [12,13]. Another obvious flaw, i.e., the absence of a half-quantized CF Hall conductance expected for a halffilled LL with the particle-hole symmetry [14], is remedied only recently by Wang et al., who show that spatial fluctuations of the effective magnetic field can induce CF scattering with side jumps and gives rise to a half-quantized CF Hall conductance [15]. 
These difficulties prompt Son to propose the Dirac CF theory [16]. Besides the aforementioned issues, Son also notices that the HLR theory does not exhibit explicitly the particlehole symmetry expected for a LL in the zero-mass limit. As an effort to address the issue, Son conjectures that CFs are massless Dirac particles. The interpretation of CFs in the Dirac CF theory differs in many ways from that in the HLR theory. Most notably, a Dirac CF traversing a Fermi circle acquires a $\pi$-Berry phase, which is absent in the HLR theory. It leads to the predictions of suppression of back-scattering of CFs [17] and an extra phase in SdH oscillations [18]. Moreover, the theory shows the swap of the notions of density and magnetic field, i.e., the density of Dirac CFs is set by the magnetic field, while the effective magnetic field felt by CFs is set by the electron density. This is because Dirac CFs are actually particles dual to electrons in a charge-neutral massless Dirac cone [19]. This is distinct from the HLR theory in which CFs remain electron-like, having the same density, Fermi wave vector and dynamics.

One of manifest distinctions between the two effective theories is in how the conductivity tensor of electrons $\sigma(\omega)$ is related to that of CFs $\tilde{\sigma}(\omega)$. In the HLR theory, the two conductivity tensors are related by a simple resistivity-shifting rule $[2,8]$ :

$$
\sigma^{-1}(\omega)=\tilde{\sigma}^{-1}(\omega)+\sigma_{\mathrm{CS}}^{-1}
$$

with

$$
\sigma_{\mathrm{CS}}=\frac{e^{2}}{2 h}\left(\begin{array}{cc}
0 & 1 \\
-1 & 0
\end{array}\right),
$$

whereas in the Dirac CF theory, they are related by [16]

$$
\sigma(\omega)=-\sigma_{\mathrm{CS}} \tilde{\sigma}^{-1}(\omega) \sigma_{\mathrm{CS}}+\sigma_{\mathrm{CS}} \beta(\omega)
$$

with

$$
\beta(\omega)=\left(\begin{array}{cc}
1 & -\mathrm{i} \omega / \tilde{\omega}_{B} \\
\mathrm{i} \omega / \tilde{\omega}_{B} & 1
\end{array}\right),
$$

where $\tilde{\omega}_{B} \equiv e B / m^{*}$ is a characteristic frequency set by the external magnetic field $\boldsymbol{B}=-B \hat{z}$. The relation is established in the long-wavelength limit, and depends on a CF effective mass parameter $m^{*}$, which is phenomenologically introduced to recover the Galilean invariance for the Dirac $\mathrm{CF}$ theory $[16,20]$. As a result of the distinction, the two theories will predict different electromagnetic (EM) responses of $\mathrm{CF}$ systems.

An alternative effective description parallel to these theories is the dipole picture proposed by Read [21]. In the picture, a $\mathrm{CF}$ is not a point particle but a dipole consisting of an electron and a double $2 \mathrm{~h} / \mathrm{e}$ quantum vortex, which is spatially separated from the electron. Intriguingly, the momentum of a $\mathrm{CF}$, which is proportional to its velocity, can be interpreted as the transverse of its dipole vector. The picture emerges naturally from the Rezayi-Read wave function $\Psi\left(\left\{z_{a}\right\}\right) \propto J\left(\left\{z_{a}+\mathrm{i} k_{a} l_{\mathrm{B}}^{2}\right\}\right) \exp \left(\mathrm{i} \sum_{a} \bar{k}_{a} z_{a} / 2\right)$, which is obtained by applying the CF ansatz $\Psi\left(\left\{z_{a}\right\}=\right.$ $\hat{P}_{\mathrm{LLL}} J\left(\left\{z_{a}\right\}\right) \psi_{\mathrm{CF}}\left(\left\{\boldsymbol{r}_{a}\right\}\right)$ to a CF wave function $\psi_{\mathrm{CF}}\left(\left\{\boldsymbol{r}_{a}\right\}\right)=$ $\exp \left(\mathrm{i} \sum_{a} \boldsymbol{k}_{a} \cdot \boldsymbol{r}_{a}\right)$ for a set of free CFs with wave vectors $\left\{\boldsymbol{k}_{a}\right\}$ in the hidden Hilbert space [22], where we denote $z_{a}=$ $x_{a}+\mathrm{i} y_{a}$ for $\boldsymbol{r}_{a} \equiv\left(x_{a}, y_{a}\right), k_{a}=k_{a x}+\mathrm{i} k_{a y}$ for $\boldsymbol{k}_{a} \equiv\left(k_{a x}, k_{a y}\right)$ and $\bar{k}_{a}$ the complex conjugate of $k_{a}, J\left(\left\{z_{a}\right\}\right) \propto \prod_{a<b}\left(z_{a}-\right.$ $\left.z_{b}\right)^{2}$ is the Bijl-Jastrow factor, $l_{B}=\sqrt{\hbar / e B}$ is the magnetic length, and $\hat{P}_{\mathrm{LLL}}$ is the projection to the lowest LL $[2,3]$. By interpreting zeros in the Bijl-Jastrow factor as quantum vortices, one observes that a double-vortex is displaced from the electron to which it is bound by $z_{a} \rightarrow z_{a}+$ $\mathrm{i} k_{a} l_{B}^{2}$. Microscopic wave functions prescribed by the theory of CFs are well tested and widely accepted as precise descriptions of many-body states of fractionally filled LLs [23]. The fact that the dipole picture is directly inferred from a microscopic wave function distinguishes it from the HLR theory and the Dirac CF theory, both of which are based on conjectured effective field theories and cannot be directly associated with actual microscopic wave functions except for a few special cases [24-26].

For a long time, the dynamics of CFs as dipoles is not explicitly specified. There is even a misunderstanding that the dipole picture is just a complement to the HLR theory and has the same dynamics. To this end, Shi and Ji derive the semiclassical dynamics of CFs in a CF Wigner crystal that is basically a set of CF wave packets in the hidden Hilbert space [27]. From the semiclassical dynamics, they obtain a dipole picture similar to that suggested by Read. Specifically, in the picture, CS-like fields emerge as a result of the correlations embodied in the Bijl-Jastrow factor and couple only to vortices, while external EM fields couple only to electrons. By adopting the same interpretation of the momentum as in Read's theory, they obtain a dynamics in which CFs are subject to a uniform Berry curvature $\Omega_{z}=1 / e B$ in the momentum space [28,29]. The presence of the Berry curvature is a clear indication that a dipole is not identical to the CF envisioned in the HLR theory.

Notably, even though the dipole picture and the Dirac CF theory are very different microscopically, their CFs share some basic properties (see Sec. II B): (1) a CF traversing a Fermi circle acquires a $\pi$-Berry phase; (2) the Fermi wave vector $k_{\mathrm{F}}=\sqrt{e B / \hbar}$ is set only by the magnetic field and independent of the electron density. These properties underly various effects predicted for the Dirac CF theory $[17,18,30]$. On the other hand, different from the Dirac CF theory, the dipole picture does not exhibit explicitly the particle-hole symmetry. It is due to the lack of the particle-hole symmetry of the vacuum assumed in the picture: the vacuum is an empty LL for electrons and a filled LL for holes. The latter has a nonzero Chern number $C_{\mathrm{vac}}=1$. The difference is superficial and may not have a physical consequence (see Sec. VIB). Therefore, it is reasonable to believe that although the two pictures are distinct microscopically, they may share predictions for macroscopic effects, e.g., the EM response.

In this paper, we determine the EM response of CFs in the dipole picture. We show that, in the long-wavelength limit, the response has a form identical to Eq. (3) of the Dirac CF theory, and is different from Eq. (1) even though the dipole picture is closer microscopically to the HLR theory. The Dirac CF-like EM response arises because the Berry curvature contributes a half-quantized intrinsic Hall conductance, which, notably, is independent of the filling factor. We show that CFs undergo no side jumps when scattered in a LL with the particle-hole symmetry. Therefore, the intrinsic contribution due to the Berry curvature is not altered by extrinsic impurity scattering. The remainder of the response is from an effective system with a Fermi wave vector $k_{\mathrm{F}}=\sqrt{e B / \hbar}$ and 
a $\pi$-Berry phase, indistinguishable from a Dirac CF system in the long-wavelength limit. By redefining the vacuum of the dipole picture and interpreting the intrinsic response as a contribution from the vacuum, we can explicitly show the emergence of a Dirac CF effective description. On the other hand, although we can reproduce the result by Wang et al. at half filling [15], the dipole theory and the HLR theory are in general not equivalent when the filling factor of a LL deviates from $1 / 2$. Finally, we show that deviations from the Dirac CF theory arise when higher order corrections due to electric quadrupoles and magnetic moments of CFs are considered.

The remainder of the paper is organized as follows. In Sec. II, we introduce the dipole picture of CFs, and discuss its dynamics as well as a constraint imposed by the particle-hole symmetry. In Sec. III, we determine the intrinsic response of CFs, and show that it contributes a half quantized Hall conductance independent of the filling factor. In Sec. IV, we show that CFs in a particle-hole symmetric LL undergo no side-jumps when scattered. Therefore, the intrinsic response is not altered by the scattering. In Sec. V, we solve the Boltzmann equation and determine the extrinsic response of CFs. In Sec. VI, we apply CS self-consistent conditions and determine the total response of CFs to EM fields, and show the emergence of the Dirac CF effective theory in the dipole picture. In Sec. VII, we determine corrections due to electric quadrupoles and magnetic moments of CFs. In Sec. VIII, we test our theory by fitting to experimental data. Finally, we summarize and discuss our results in Sec. IX.

\section{DIPOLE PICTURE OF CFs}

\section{A. Lagrangian}

In the dipole picture, a CF is the bound state of an electron and a double quantum vortex. The electron and the vortex are separated spatially, and bounded together by an attractive force due to the void created by the vortex and the repulsive Coulomb interaction between electrons [21]. CS-like fields emerge and couple to vortices. Meanwhile, electrons are coupled to external EM fields including the strong magnetic field, which confines electrons in a LL. This is the dipole picture explicated in Ref. [27]. Our study will be based on this particular dipole picture instead of other variants, which can be found in literatures [31-37].

The picture of CFs is described by the action in $(2+1)$ dimensions:

$$
S=\int d^{3} x\left(-A \cdot j^{\mathrm{e}}-a \cdot j+\frac{e^{2}}{4 h} \epsilon^{\mu \nu \gamma} a_{\mu} \partial_{\nu} a_{\gamma}-\varepsilon\right),
$$

where $\left(j^{\mathrm{e}}\right)^{\mu} \equiv\left(\rho^{\mathrm{e}}, \boldsymbol{j}^{\mathrm{e}}\right)$ and $j^{\mu} \equiv(\rho, \boldsymbol{j})$ are charge-current densities of electrons and vortices, $A^{\mu} \equiv(\Phi, \boldsymbol{A})$ and $a^{\mu} \equiv$ $(\phi, \boldsymbol{a})$ denote the external EM fields and the emergent CS fields, respectively, and $\varepsilon$ denotes the binding energy between electrons and quantum vortices. The action assumes that both electrons and vortices have zero mass and are subject to the external magnetic field and the CS magnetic field, respectively. As a result, the motion of the electrons (vortices) is confined in a LL created by the external (CS) magnetic field.
Under the mean-field approximation, differentiating the action with respect to the CS fields $a$ gives rise to the selfconsistent conditions

$$
\begin{gathered}
\rho=-\frac{e^{2}}{2 h} b, \\
\boldsymbol{j}=-\sigma_{\mathrm{cs}} \boldsymbol{e},
\end{gathered}
$$

where $\boldsymbol{e}=-\partial_{t} \boldsymbol{a}-\nabla \boldsymbol{\phi}$ and $\boldsymbol{b}=\boldsymbol{\nabla} \times \boldsymbol{a} \equiv b \hat{z}$ are CS electric and magnetic fields, respectively. While the HLR theory has the same set of self-consistent conditions, the dipole picture differs in that (a) the CS fields only couple to vortices and are not equivalent in effects to the EM fields; (b) the conditions as well as the CS term in Eq. (5) should be regarded as an approximation only for the long-wavelength limit. Corrections are expected when the wavelength of the EM fields is comparable to the length scale of the dipoles, i.e., the magnetic length $l_{\mathrm{B}}$ [27].

To proceed, we relate the charge-current densities of electrons $j^{\mathrm{e}}$ to their counterparts for vortices $j$. The charge-current densities of electrons are defined by

$$
\begin{gathered}
\rho^{\mathrm{e}}(t, \boldsymbol{x})=-e \sum_{a} \delta\left(\boldsymbol{x}-\boldsymbol{x}_{a}^{\mathrm{e}}(t)\right), \\
\boldsymbol{j}^{\mathrm{e}}(t, \boldsymbol{x})=-e \sum_{a} \dot{\boldsymbol{x}}_{a}^{\mathrm{e}} \delta\left(\boldsymbol{x}-\boldsymbol{x}_{a}^{\mathrm{e}}(t)\right),
\end{gathered}
$$

where $\left\{\boldsymbol{x}_{a}^{\mathrm{e}}(t)\right\}$ denotes the set of electron coordinates. The charge-current densities for vortices $(\rho, \boldsymbol{j})$ can be similarly defined by using the set of vortex coordinates $\left\{\boldsymbol{x}_{a}(t)\right\}$. We introduce the dipole vector

$$
\boldsymbol{d}_{a} \equiv \boldsymbol{x}_{a}^{\mathrm{e}}-\boldsymbol{x}_{a} .
$$

After applying multipole expansions [38], we obtain

$$
\begin{gathered}
\rho^{\mathrm{e}}=\rho-\nabla \cdot \boldsymbol{P}+\nabla \nabla: \mathbb{Q} \ldots, \\
\boldsymbol{j}^{\mathrm{e}}=\boldsymbol{j}+\partial_{t}(\boldsymbol{P}-\nabla \cdot \mathbb{Q})+\nabla \times \boldsymbol{M}+\ldots,
\end{gathered}
$$

where we keep dipole and quadrupole corrections for the charge density, and displacement current and magnetic dipole corrections for the current density. Because CFs have a length scale $l_{B}$ and an energy scale $\hbar \tilde{\omega}_{B}$, the expansions are in rising orders of $q l_{B}$ and $\omega / \tilde{\omega}_{B}$, where $\omega$ and $q$ are the frequency and wavenumber of probing EM fields, respectively. The dipole density $\boldsymbol{P}$, the quadrupole density tensor $\mathbb{Q}$, and the magnetization density $\boldsymbol{M}$ are defined, respectively, by

$$
\begin{gathered}
\boldsymbol{P}(t, \boldsymbol{x})=-e \sum_{a} \boldsymbol{d}_{a} \delta\left(\boldsymbol{x}-\boldsymbol{x}_{a}\right), \\
\mathbb{Q}(t, \boldsymbol{x})=-\frac{e}{2} \sum_{n} \boldsymbol{d}_{a} \boldsymbol{d}_{a} \delta\left(\boldsymbol{x}-\boldsymbol{x}_{a}\right), \\
\boldsymbol{M}(t, \boldsymbol{x})=-e \sum_{a} \boldsymbol{d}_{a} \times\left(\dot{\boldsymbol{x}}_{a}+\frac{1}{2} \dot{\boldsymbol{d}}_{a}\right) \delta\left(\boldsymbol{x}-\boldsymbol{x}_{a}\right) .
\end{gathered}
$$

Substituting the expansions into Eq. (5) and applying integrals by parts, we obtain

$$
\begin{aligned}
S \approx \int d^{2} \boldsymbol{x} d t[ & -(a+A) \cdot j+\frac{e^{2}}{4 h} \epsilon^{\mu \nu \gamma} a_{\mu} \partial_{\nu} a_{\gamma} \\
& +\boldsymbol{M} \cdot \boldsymbol{B}-(\varepsilon-\boldsymbol{E} \cdot \boldsymbol{P}-\mathbb{Q}: \nabla \boldsymbol{E})]
\end{aligned}
$$


We then follow Read to define the momentum of a $\mathrm{CF}$ as [21]

$$
\boldsymbol{p}_{a}=e B \hat{z} \times \boldsymbol{d}_{a} .
$$

We find

$$
\boldsymbol{M} \cdot \boldsymbol{B}=\sum_{a} \boldsymbol{p}_{a} \cdot \dot{\boldsymbol{x}}_{a}+\frac{1}{2 e B}\left(\boldsymbol{p}_{a} \times \dot{\boldsymbol{p}}_{a}\right) \cdot \hat{z} .
$$

Substituting it into Eq. (16) and interpreting $\boldsymbol{x}_{a}$ as the coordinate of a CF, we obtain an action in which a CF is governed by the Lagrangian

$$
\begin{aligned}
L_{a}= & \boldsymbol{p}_{a} \cdot \dot{\boldsymbol{x}}_{a}+\frac{1}{2 e B}\left(\boldsymbol{p}_{a} \times \dot{\boldsymbol{p}}_{a}\right) \cdot \hat{z}-e \dot{\boldsymbol{x}}_{a} \cdot \tilde{\boldsymbol{a}}\left(t, \boldsymbol{x}_{a}\right) \\
& +e \tilde{\phi}\left(t, \boldsymbol{x}_{a}\right)-\mathcal{E}_{a} .
\end{aligned}
$$

From the Lagrangian, we see that the $\mathrm{CF}$ is subject to an effective field $\tilde{a} \equiv(\tilde{\phi}, \tilde{\boldsymbol{a}})=a+A$, a Berry curvature $\Omega_{z}=1 / e B$, and an energy dispersion with electric dipole and quadrupole corrections

$$
\begin{aligned}
\mathcal{E}_{a}= & \varepsilon\left(\boldsymbol{p}_{a}\right)-\frac{1}{B}(\boldsymbol{E} \times \hat{z}) \cdot \boldsymbol{p}_{a} \\
& +\frac{1}{2 e B^{2}}\left(\boldsymbol{p}_{a} \times \hat{z}\right) \cdot \nabla \boldsymbol{E} \cdot\left(\boldsymbol{p}_{a} \times \hat{z}\right),
\end{aligned}
$$

where $\varepsilon\left(\boldsymbol{p}_{a}\right)$ denotes the binding energy of a $\mathrm{CF}$, and $\varepsilon=$ $\sum_{a} \varepsilon\left(\boldsymbol{p}_{a}\right)$.

We model the binding energy as a harmonic potential $\varepsilon\left(\boldsymbol{p}_{a}\right) \propto\left|\boldsymbol{d}_{a}\right|^{2}$. After substituting Eq. (17), the binding energy becomes the dispersion of CFs, and can be written as

$$
\varepsilon\left(\boldsymbol{p}_{a}\right)=\frac{b}{B} \frac{p_{a}^{2}}{2 m^{*}},
$$

where we interpret $m^{*}$ as the effective mass of CFs. For a reason, which will be clarified in Sec. IIC, we append a $b / B$ factor to the dispersion. The extra factor becomes 1 at half filling.

\section{B. Dynamics}

From the Lagrangian Eq. (19), we can obtain equations of motion of CFs. For the moment, we focus on the longwavelength limit and ignore all spatial gradients of $\boldsymbol{E}, \boldsymbol{B}$, and $\boldsymbol{b}$. The time dependence of $\boldsymbol{B}$ is also ignored since $\partial_{t} \boldsymbol{B}=$ $-\boldsymbol{\nabla} \times \boldsymbol{E}$ is of the same order of the gradient of $\boldsymbol{E}$. The equations read:

$$
\begin{gathered}
\dot{\boldsymbol{x}}=\frac{b}{B} \frac{\boldsymbol{p}}{m^{*}}-\frac{1}{B} \boldsymbol{E} \times \hat{z}-\frac{1}{e B} \dot{\boldsymbol{p}} \times \hat{z}, \\
\dot{\boldsymbol{p}}=-e \tilde{\boldsymbol{e}}-e \tilde{b} \dot{\boldsymbol{x}} \times \hat{z},
\end{gathered}
$$

where for the simplicity of notations we drop the subscripts indexing particles, $\tilde{\boldsymbol{e}}=\boldsymbol{e}+\boldsymbol{E}$ and $\tilde{b}=b-B$ are the effective electric and magnetic fields experienced by $\mathrm{CFs}$, respectively. The equations should be regarded as a zeroth order approximation. Corrections due to gradients of the EM and CS fields will be determined in Sec. VII.

The dynamics of CFs in the dipole picture is different from that assumed in the HLR theory. Two differences are notable: (a) CFs are subject to a Berry curvature $\Omega_{z}=1 / e B$; (b) The external electric field $\boldsymbol{E}$ introduces a correction to the group velocity due to the dipole correction to the energy. In the HLR theory, EM fields can be completely absorbed into the effective fields. Therefore, they are equivalent to the CS fields in driving CFs. This is not true anymore in the dipole picture. Besides being a part of the effective fields, they also introduce the Berry curvature and the dipole correction. As we will see, these corrections change fundamentally how a $\mathrm{CF}$ system responses to EM fields.

The presence of the Berry curvature has important physical consequences. It introduces a $\pi$-Berry phase for an electron traversing a Fermi circle. The $\pi$-Berry phase is regarded as a feature of the Dirac CF theory, and its effects has been extensively explored $[17,18]$. Another consequence of the Berry curvature is a correction to the phase-space density-of-states [39]. In ordinary two-dimensional systems, a quantum state always occupies a $h^{2}$ phase-space volume. In the presences of both the Berry curvature and the (effective) magnetic field, however, the phase space volume element $d^{2} x d^{2} p / h^{2}$ is modified to $D d^{2} x d^{2} p / h^{2}$ by the density-of-state correction factor

$$
D=1+e \tilde{b} \Omega_{z}=\frac{b}{B} .
$$

For a homogeneous system, we have $D=2 v$, where $v \equiv$ $-\rho^{\mathrm{e}} h / e^{2} B$ is the filling factor of the system. The factor modifies the Fermi wave vector from its usual value $k_{\mathrm{F}}^{0}=\sqrt{4 \pi n}$, where $n$ is the density of CFs, to

$$
k_{\mathrm{F}}=k_{\mathrm{F}}^{0} / \sqrt{D}=\sqrt{\frac{e B}{\hbar}} .
$$

We see that, even though the CF density in the dipole picture is the same as the electron density, the Fermi wave vector is set only by the magnetic field $B$ and independent of the density. The prediction differs from the HLR theory and agrees with the Dirac CF theory but has a different interpretation: $k_{\mathrm{F}}$ is modified due not to a change of the CF density but the phasespace density-of-state correction.

For the convenience of later applications, the equations of motion Eqs. (22) and (23) can be solved for $\dot{\boldsymbol{x}}$ and $\dot{\boldsymbol{p}}$ :

$$
\begin{gathered}
\dot{\boldsymbol{x}}=\frac{\boldsymbol{p}}{m^{*}}-\frac{1}{b} \hat{z} \times \boldsymbol{e}, \\
\dot{\boldsymbol{p}}=e \tilde{b} \hat{z} \times \frac{\boldsymbol{p}}{m^{*}}-\frac{1}{D} e \boldsymbol{e}-e \boldsymbol{E} .
\end{gathered}
$$

\section{Particle-hole symmetry and CF dispersion}

The particle-hole symmetry imposes a constraint on how the $\mathrm{CF}$ dispersion depends on the filling factor. To see that, we assume that CFs have the dispersion $\varepsilon_{p}=\boldsymbol{p}^{2} / 2 m^{\prime}$ as in the HLR theory. Away from half-filling, CFs experience an effective magnetic field $\tilde{b}$, and form $\Lambda$-levels [2]. In our case, the gap between neighboring $\Lambda$-levels is $[39,40]$

$$
\Delta=\frac{\hbar e|\tilde{b}|}{D m^{\prime}} .
$$

The presence of $D$ in the denominator is notable. The particlehole symmetry requires that the gap at fillings $v$ and $1-v$ should be equal when one fixes the external magnetic field and changes the density [2]. However, if $m^{\prime}(1-v)=m^{\prime}(v)$ is assumed as in the HLR theory, the gap predicted by Eq. (28) 
will be asymmetric for particles and holes since $D=b / B=$ $2 v$ is proportional to the filling factor.

To compensate, we explicitly include a factor $D$ to the $\mathrm{CF}$ dispersion in Eq. (21), and interpret $m^{*}$ as the effective mass of CFs. With the factor, the particle-hole symmetry requires

$$
m^{*}(v)=m^{*}(1-v) .
$$

We note that $m^{*}$ also depends on $B$, as shown in Ref. [8].

\section{INTRINSIC RESPONSE}

\section{A. Intrinsic Hall conductance}

We first determine the intrinsic response, which is the EM response when the system is in a local equilibrium state. In our case, the distribution function of the local equilibrium state is

$$
f_{0}(\boldsymbol{x}, \boldsymbol{p}, t)=n_{\mathrm{F}}(\mathcal{E}(\boldsymbol{p})-\mu),
$$

where $n_{\mathrm{F}}$ is the Fermi-Dirac distribution function, $\mu$ is the chemical potential, and $\mathcal{E}(\boldsymbol{p})$ is the energy dispersion shown in Eq. (20). The intrinsic contribution to the current is

$$
\boldsymbol{j}_{0}=-e \int[d \boldsymbol{p}] D \dot{\boldsymbol{x}} f_{0}(\boldsymbol{p})
$$

with $[d \boldsymbol{p}] \equiv d^{2} p / h^{2}$.

To evaluate the intrinsic current, we note that the electric field is to shift the center of the $\mathrm{CF}$ dispersion, i.e., $\mathcal{E}(\boldsymbol{p}) \approx \varepsilon\left(\boldsymbol{p}-m^{*} \boldsymbol{E} \times \hat{z} / b\right)$ to the linear order of $\boldsymbol{E}$. Substituting Eq. (26) and the dispersion into Eq. (31), and making the substitution $\boldsymbol{p} \rightarrow \boldsymbol{p}+m^{*} \boldsymbol{E} \times \hat{z} / b$, we have

$$
\boldsymbol{j}_{0}=-e \int[d \boldsymbol{p}] D n_{\mathrm{F}}(\varepsilon(\boldsymbol{p})-\mu)\left(\frac{\boldsymbol{p}}{m^{*}}-\frac{\hat{z} \times \tilde{\boldsymbol{e}}}{b}\right) .
$$

The first term of the integral vanishes, and the second term can be determined by using the identity $-e \int[d \boldsymbol{p}] D n_{\mathrm{F}}(\varepsilon(\boldsymbol{p})-$ $\mu)=\rho$ and the self-consistent condition Eq. (6). We obtain

$$
\boldsymbol{j}_{0}=-\sigma_{\mathrm{CS}} \tilde{\boldsymbol{e}} .
$$

The intrinsic response contributes a Hall conductance $\tilde{\sigma}_{x y}^{0}=-e^{2} / 2 h$ for the effective electric field. It is notable that the Hall conductance is independent of the density or the filling factor.

\section{B. Definition of the CF coordinate}

In the last subsection, we obtain a half-quantized intrinsic Hall conductance for CFs. It seems to provide a solution to the issue raised by Kivelson et al., i.e., CFs must have a halfquantized Hall conductance in a particle-hole symmetric LL [14]. However, the issue is not fully resolved due to an ambiguity in defining the coordinate of a CF. The interpretation of the dynamics, in particular the presence of a Berry curvature, depends on the definition we adopt for the CF coordinate, while the intrinsic Hall conductance is directly related to the Berry curvature.

To see that, we examine the Lagrangian Eq. (19) to see how the definition of the CF coordinate affects its interpretation. At half-filling, the Lagrangian for a $\mathrm{CF}$ has the form $L=\boldsymbol{p} \cdot \dot{\boldsymbol{x}}+(\boldsymbol{p} \times \dot{\boldsymbol{p}}) \cdot \hat{z} / 2 e B+e \tilde{\phi}(\boldsymbol{x})-\mathcal{E}$, where $\boldsymbol{x}$ is the coordinate of the vortex in the CF. We implicitly define the coordinate of the $\mathrm{CF}$ as the coordinate of its vortex and interpret the second term as a Berry curvature $\Omega_{z}=1 / e B$. However, the interpretation changes with an alternative definition of the $\mathrm{CF}$ coordinate. For instance, if we define the $\mathrm{CF}$ coordinate as the center coordinate $\boldsymbol{x}^{\mathrm{c}}=\boldsymbol{x}+\boldsymbol{d} / 2=\boldsymbol{x}+\boldsymbol{p} \times$ $\hat{z} / 2 e B$, the Lagrangian becomes $L=\boldsymbol{p} \cdot \dot{\boldsymbol{x}}^{\mathrm{c}}+e \tilde{\phi}(\boldsymbol{x})-\mathcal{E}$, and the Berry curvature vanishes. Similarly, if we define the CF coordinate as the coordinate of its electron, the Berry curvature will reverse its sign. Then, what would be the physical interpretation?

The answer lies in the local equilibrium state that the system adapts to. When external fields are applied, the system rapidly relaxes to a local equilibrium state with its momentum distribution determined by momentum relaxation processes. By assuming the local equilibrium distribution Eq. (30), we implicitly assume that the momentum relaxation processes do not change $\boldsymbol{x}$, i.e., the position of the vortex, otherwise the effective potential $\tilde{\phi}(\boldsymbol{x})$ would affect the energy conservation of relaxation processes and therefore the detailed balance condition for determining the local equilibrium distribution. It is the momentum relaxation process instead of an arbitrary choice of the definition of the CF coordinate that determines the intrinsic response.

To elucidate the point, we try an alternative definition of the $\mathrm{CF}$ coordinate, say, the center of the dipole $\boldsymbol{x}^{\mathrm{c}}$. In this case, multipole expansions lead to a dispersion $\mathcal{E}^{\prime}(\boldsymbol{p})=\varepsilon(\boldsymbol{p})-$ $[(\boldsymbol{E}-\boldsymbol{e}) \times \hat{z}] \cdot \boldsymbol{p} / 2 B$. We still assume that a momentum relaxation process does not change the position of the vortex. It implies that a process inducing a momentum change $\Delta p$ also induces a change of the $\mathrm{CF}$ coordinate (side jump) $\Delta \boldsymbol{x}^{\mathrm{c}}=$ $\Delta \boldsymbol{p} \times \hat{z} / 2 e B$, and a change of energy $\Delta \mathcal{E}^{\prime}(\boldsymbol{p})+e(\boldsymbol{e}+\boldsymbol{E})$. $\Delta \boldsymbol{x}^{\mathrm{c}}=\Delta \varepsilon(\boldsymbol{p})-(\boldsymbol{E} \times \hat{z}) \cdot \Delta \boldsymbol{p} / B \equiv \Delta \mathcal{E}(\boldsymbol{p})$. The principle of detailed balance will lead to the same local equilibrium distribution Eq. (30).

In the next section, we will establish that the momentum relaxation processes in a LL with the particle-hole symmetry do keep $\boldsymbol{x}$ unchanged. It makes $\boldsymbol{x}$ a convenient choice as the CF coordinate since one needs not to deal with the complexities associated with side jumps [15]. When the filling factor deviates from $1 / 2$, there is another reason for choosing $x$ as the $\mathrm{CF}$ coordinate. In this case, the particular choice provides the simplest form of the equations of motions. With other choices, nondiagonal Berry curvature components will appear, making the equations more complicated and their interpretation more difficult. The latter point is discussed in Ref. [27].

\section{SCATTERING OF CFs}

In this section, we investigate the momentum relaxation processes of CFs. At low temperatures, the processes are dominated by elastic scattering induced by quenched impurities. For CF systems, there are two possible ways that impurities can affect CFs: (a) impurities induce a random potential coupling to the electrons in dipoles; (b) the random impurity potential induces a spatial modulation of the CF density, and in turn induces a spatially fluctuating CS magnetic field. We will show that CFs undergo no side-jumps when scattered by both the fields as long as CFs adopt a dispersion consistent to the particle-hole symmetry requirement Eq. (29). It justifies our choice of defining the coordinate of a $\mathrm{CF}$ as its vortex position. 
$\boldsymbol{B} \otimes$
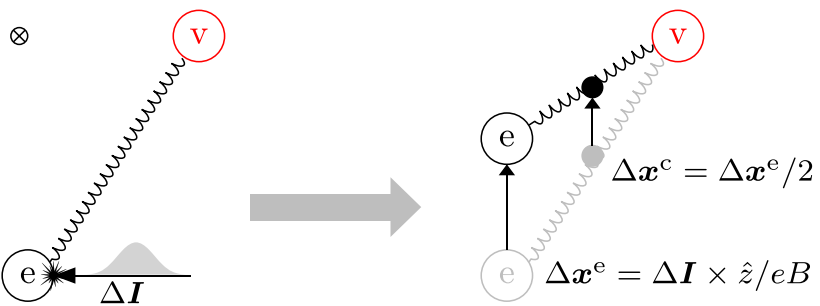

FIG. 1. Collision between a CF and an impurity. Left: the impurity exerts an impulse $\Delta \boldsymbol{I}$ to the electron in the CF. Right: after the collision, the electron coordinate as well as the center coordinate $\boldsymbol{x}^{\mathrm{c}}$ are shifted. Depending on the definition of the CF coordinate as the center coordinate $\boldsymbol{x}^{\mathrm{c}}$ or the vortex coordinate $\boldsymbol{x}$, the scattering can be interpreted as with or without a side jump, respectively.

We will first consider scattering by the impurity potential only. The more complete consideration including the fluctuating CS field will be presented in Sec. IV B. Our consideration is similar to Wang et al.'s consideration for the HLR theory [15]. The correspondence between the two considerations will be discussed in Sec. IVC. Our result also coincides with numerical observations by Geraedts et al. [41]. It will be discussed in Sec. IV D.

\section{A. Scattering by the impurity potential}

At low temperatures, the momentum relaxation is dominated by elastic scattering induced by impurities ubiquitously presented in real materials. Impurities introduce a random potential into the system. As an electrostatic potential, it only couples to the electrons in dipoles. Electrons feel an effective random potential $V_{\text {eff }}\left(\boldsymbol{x}^{\mathrm{e}}\right)$, which consists of the random potential imposed directly by impurities and a screening potential from other electrons. It is important to note that the potential depends on $\boldsymbol{x}^{\mathrm{e}}$ instead of $\boldsymbol{x}$.

We first present a heuristic picture for a CF colliding with an impurity. We assume that the scattering is instantaneous, i.e., the impurity exerts an impulsive force to the electron with a total impulse $\Delta \boldsymbol{I}$. The motion of an electron confined in a LL is governed by the equation $e B \hat{z} \times \dot{\boldsymbol{x}}^{\mathrm{e}}=\boldsymbol{F}$, where $\boldsymbol{F}$ is the total force acting on the electron, including the binding force from the vortex and the impulsive force exerted by the impurity. Since the binding force is negligible in the infinitesimal time period of the collision, the equation predicts that the electron will have a coordinate shift $\Delta \boldsymbol{x}^{\mathrm{e}}=\Delta \boldsymbol{I} \times \hat{z} / e B$ after the collision. Meanwhile, the position of the vortex does not change. The process of the collision is illustrated in Fig. 1. According to the dipole picture and Eq. (17), it corresponds to a CF momentum change $\Delta \boldsymbol{p}=e B \hat{z} \times \Delta \boldsymbol{x}^{\mathrm{e}}=\Delta \boldsymbol{I}$ and no change to the $\mathrm{CF}$ coordinate. We therefore conclude that the collision does not induce a side jump. Note that the conclusion is valid only when we define the coordinate of the $\mathrm{CF}$ as its vortex position. Had we chosen $\boldsymbol{x}^{\mathrm{c}}$ as the CF coordinate, for instance, we would conclude that the collision induces a side jump $\Delta \boldsymbol{x}^{\mathrm{c}}=\Delta \boldsymbol{I} \times \hat{z} / 2 e \boldsymbol{B}=\Delta \boldsymbol{p} \times \hat{z} / 2 e B$.

To treat the problem more formally, we follow the approach developed by Sinitsyn et al. [42]. For a canonical quantum system, it is shown that the side jump induced by elastic scattering is

$$
\Delta \tilde{\boldsymbol{x}}_{\tilde{p}^{\prime}, \tilde{p}}=-\hbar\left(\partial_{\tilde{p}}+\partial_{\tilde{p}^{\prime}}\right) \arg \left[V_{\tilde{p}^{\prime}, \tilde{p}}\right],
$$

where $V_{\tilde{\boldsymbol{p}}^{\prime}, \tilde{\boldsymbol{p}}}=\left\langle\tilde{\boldsymbol{p}}^{\prime}|\hat{V}| \tilde{\boldsymbol{p}}\right\rangle$ is the matrix element of a scattering potential $\hat{V}$ in the momentum eigenstates $|\tilde{\boldsymbol{p}}\rangle$ and $\left\langle\tilde{\boldsymbol{p}}^{\prime}\right|$, and $\arg \left[V_{\tilde{p}^{\prime}, \tilde{p}}\right]$ denotes its phase angle. Note that the formula is valid for a canonical system, i.e., its classical Lagrangian should have the form of $L=\tilde{\boldsymbol{p}} \cdot \dot{\tilde{\boldsymbol{x}}}-H(\tilde{\boldsymbol{x}}, \tilde{\boldsymbol{p}})$, where $\tilde{\boldsymbol{x}}$ and $\tilde{\boldsymbol{p}}$ are the canonical coordinate and momentum, respectively.

For more general Lagrangians in which dynamic variables are not necessarily canonical, the side jump can be determined by the following procedure: (a) find a transformation from original dynamical variables $(\boldsymbol{x}, \boldsymbol{p})$ to canonical variables $(\tilde{\boldsymbol{x}}, \tilde{\boldsymbol{p}}) ;(\mathrm{b})$ determine the scattering matrix element and the side jump $\Delta \tilde{\boldsymbol{x}}_{\tilde{\boldsymbol{p}}^{\prime}, \tilde{p}}$ by using Eq. (34); (c) determine the side jump in terms of $(\boldsymbol{x}, \boldsymbol{p})$ by transforming the canonical variables back to the original dynamic variables.

We can determine the side jump for the current case by applying the procedure. At half filling, the Lagrangian Eq. (19) is reduced to $L=\boldsymbol{p} \cdot \dot{\boldsymbol{x}}+(\boldsymbol{p} \times \dot{\boldsymbol{p}}) \cdot \hat{z} / 2 e B-\varepsilon-V_{\text {eff }}$. It is easy to find the canonical variables $\tilde{\boldsymbol{p}}=\boldsymbol{p}$ and $\tilde{\boldsymbol{x}}=\boldsymbol{x}^{\mathrm{c}}=\boldsymbol{x}+\boldsymbol{p} \times$ $\hat{z} / 2 e B$. Using the canonical variables, the scattering potential can be written as $V_{\text {eff }}\left(\boldsymbol{x}^{\mathrm{e}}\right)=V_{\text {eff }}(\tilde{\boldsymbol{x}}+\tilde{\boldsymbol{p}} \times \hat{z} / 2 e B)$. The scattering matrix element can be determined straightforwardly:

$$
V_{\tilde{\boldsymbol{p}}^{\prime}, \tilde{\boldsymbol{p}}}=\tilde{V}_{\mathrm{eff}}(\boldsymbol{q}) \exp \left[\mathrm{i} \frac{\left(\tilde{\boldsymbol{p}}^{\prime} \times \tilde{\boldsymbol{p}}\right) \cdot \hat{z}}{2 e B \hbar}\right],
$$

where $\tilde{V}_{\text {eff }}(\boldsymbol{q})$ is the Fourier transform of the effective impurity potential, and $\hbar \boldsymbol{q}=\tilde{\boldsymbol{p}}^{\prime}-\tilde{\boldsymbol{p}} \equiv \Delta \boldsymbol{p}$ is the change of the momentum induced by the scattering. By applying Eq. (34), we obtain

$$
\Delta \tilde{\boldsymbol{x}}_{\tilde{\boldsymbol{p}}^{\prime}, \tilde{\boldsymbol{p}}}=\frac{\hbar \boldsymbol{q} \times \hat{z}}{2 e B} .
$$

The result is identical to $\Delta \boldsymbol{x}^{\mathrm{c}}$ determined in the heuristic argument. Transforming back to the original variables, we have

$$
\Delta \boldsymbol{x}_{\boldsymbol{p}^{\prime}, \boldsymbol{p}}=\Delta \tilde{\boldsymbol{x}}_{\boldsymbol{p}^{\prime}, \boldsymbol{p}}-\Delta\left[\frac{\boldsymbol{p} \times \hat{z}}{2 e B}\right]=0 .
$$

It confirms our conclusion from the heuristic argument that scattering of CFs by impurities does not induce side jumps.

\section{B. Effect of the fluctuating CS magnetic field}

In the last subsection, we analyze the scattering of CFs by considering only the effective impurity potential. However, the potential can also induce a density modulation of CFs. According to Eq. (6), the density modulation will induce a spatially fluctuating CS magnetic field. Wang et al. analyze the effect of the fluctuating CS magnetic field in the HLR theory, and find that it induces side jumps and gives rise to a half-quantized CF Hall conductance [15]. Here, we consider the same effect in the dipole picture.

We first determine the modification to the Lagrangian by the fluctuating CS magnetic field. Following Wang et al.'s consideration, we can determine the fluctuating CS magnetic field $\delta b(\boldsymbol{x})=-\left(2 m^{*} / e \hbar\right) V_{\text {eff }}(\boldsymbol{x})$ [15]. It gives rise to a CS 
vector potential $\delta \boldsymbol{a}(\boldsymbol{x})$. The Lagrangian is modified to

$$
L=\boldsymbol{p} \cdot \dot{\boldsymbol{x}}+\frac{1}{2 e B}(\boldsymbol{p} \times \dot{\boldsymbol{p}}) \cdot \hat{z}-e \delta \boldsymbol{a}(\boldsymbol{x}) \cdot \dot{\boldsymbol{x}}-\varepsilon-V_{\mathrm{eff}}\left(\boldsymbol{x}^{\mathrm{e}}\right)
$$

Following the procedure outlined in the last subsection, we proceed to find a set of canonical variables $(\tilde{\boldsymbol{x}}, \tilde{\boldsymbol{p}})$ for the modified Lagrangian. Unlike the previous case, the presence of the CS vector potential makes both $\boldsymbol{x}$ and $\boldsymbol{p}$ noncanonical. As in Wang et al.'s consideration, we assume that the impurity potential is weak and slowly varying in space. It thus suffices to find a transformation accurate to the linear order of $\delta \boldsymbol{a}$ and its first spatial gradient. We have [43]

$$
\begin{gathered}
\boldsymbol{x}=\tilde{\boldsymbol{x}}+\frac{1}{2 e B} \hat{z} \times\left[\boldsymbol{p}+\frac{1}{2 B}(\hat{z} \times \boldsymbol{p}) \cdot \nabla \delta \boldsymbol{a}(\tilde{\boldsymbol{x}})\right], \\
\boldsymbol{p}=\left[1-\frac{\delta b(\tilde{\boldsymbol{x}})}{2 B}\right] \tilde{\boldsymbol{p}}+e \delta \boldsymbol{a}(\tilde{\boldsymbol{x}}) .
\end{gathered}
$$

It is straightforward to verify that $L=\tilde{\boldsymbol{p}} \cdot \dot{\tilde{\boldsymbol{x}}}-\varepsilon-V_{\text {eff }}-$ $d[\delta \boldsymbol{a}(\boldsymbol{x}) \cdot(\hat{z} \times \boldsymbol{p}) / 2 B] / d t+O\left[(\delta a)^{2}, \nabla^{2} a\right]$, i.e., $\tilde{\boldsymbol{x}}$ and $\tilde{\boldsymbol{p}}$ are indeed canonical.

We can then determine the perturbation to the hamiltonian of the canonical system. Besides the direct perturbation from $\hat{V}_{\text {eff }}\left(\hat{\boldsymbol{x}}_{\mathrm{e}}\right)$, there is a perturbation to the kinetic energy $\varepsilon(\hat{p})=$ $D \hat{p}^{2} / 2 m^{*}$ induced by the CS magnetic field. The perturbation can arise from: (a) the substitution of the momentum operator Eq. (40); (b) the modulation of $D(\boldsymbol{x}) \approx 1+\delta b(\tilde{\boldsymbol{x}}) / B$ induced by the CS magnetic field modulation; (c) the dependence of $m^{*}$ on the filling factor $v$, which is also modulated with the local density. For a particle-hole symmetric LL, the contribution from (c) disappears in the linear order because $m^{*}$ is symmetric about $v=1 / 2$. Moreover, the extra factor before $\tilde{\boldsymbol{p}}$ in Eq. (40) just cancels the contribution from (b). Therefore, the total perturbation is

$$
\hat{V}=\frac{e}{2 m^{*}}[\hat{\tilde{\boldsymbol{p}}} \cdot \delta \boldsymbol{a}(\tilde{\boldsymbol{x}})+\delta \boldsymbol{a}(\tilde{\boldsymbol{x}}) \cdot \hat{\tilde{\boldsymbol{p}}}]+\hat{V}_{\mathrm{eff}}\left(\hat{\boldsymbol{x}}_{\mathrm{e}}\right) .
$$

We proceed to determine the scattering matrix element. The Fourier transform of $\delta \boldsymbol{a}(\tilde{\boldsymbol{x}})$ is $\delta \tilde{\boldsymbol{a}}(\boldsymbol{q})=-\mathrm{i} \delta \tilde{b}(\boldsymbol{q})(\hat{z} \times$ $\left.\boldsymbol{q} / q^{2}\right)=\mathrm{i}\left(2 m^{*} / e \hbar q^{2}\right)(\hat{z} \times \boldsymbol{q}) \tilde{V}_{\text {eff }}(\boldsymbol{q})$. Thus, the total scattering matrix element is

$$
V_{\tilde{\boldsymbol{p}}^{\prime}, \tilde{\boldsymbol{p}}}=\tilde{V}_{\mathrm{eff}}(\boldsymbol{q})\left[e^{\mathrm{i} \frac{\left(\tilde{p}^{\prime} \times \tilde{p}\right) \cdot \hat{z}}{2 e B \hbar}}+\frac{2 \mathrm{i}\left(\tilde{\boldsymbol{p}}^{\prime} \times \tilde{\boldsymbol{p}}\right) \cdot \hat{z}}{\hbar^{2} q^{2}}\right] .
$$

Following Wang et al.'s assumption that $\tilde{V}_{\mathrm{eff}}(\boldsymbol{q}) / q$ does not diverge at $q \rightarrow 0$, we determine that the phase angle of the scattering matrix element is $\arg \left(V_{\tilde{\boldsymbol{p}}^{\prime}, \tilde{\boldsymbol{p}}}\right) \approx-\hbar^{2} q^{2} / 2\left[\left(\tilde{\boldsymbol{p}}^{\prime} \times \tilde{\boldsymbol{p}}\right)\right.$. $\hat{z}]$ to the linear order of $q l_{B}$.

Applying Eq. (34), we determine the side jump in the canonical system:

$$
\Delta \tilde{\boldsymbol{x}}_{\tilde{\boldsymbol{p}}^{\prime}, \tilde{\boldsymbol{p}}}=\frac{\boldsymbol{q} \times \hat{z}}{2 k_{\mathrm{F}}^{2}},
$$

for CFs on the Fermi circle. Substituting $k_{\mathrm{F}}=\sqrt{e B / \hbar}$ into the relation, we find that it is identical to Eq. (36) determined for a scalar potential.

Finally, we determine the side-jump in terms of $\boldsymbol{x}$ and $\boldsymbol{p}$. Note that the side jump is determined by comparing particle trajectories in asymptotic regions far from an impurity [42]. In these regions, the CS field $\delta \boldsymbol{a}(\boldsymbol{x})$ induced by the impurity is negligible. Therefore, the relation between $(\Delta x, \Delta p)$ and $(\Delta \tilde{\boldsymbol{x}}, \Delta \tilde{\boldsymbol{p}})$ is the same as Eq. (37). We thus have

$$
\Delta \boldsymbol{x}_{\boldsymbol{p}^{\prime}, \boldsymbol{p}}=0 \text {. }
$$

It indicates that when both the impurity potential and the fluctuating CS magnetic field are considered, the scattering of CFs does not induce side jumps in a LL with the particle-hole symmetry.

\section{Compared to Wang et al.'s result}

The discussion in the last subsection closely follows Wang et al.'s model on how impurities affect a CF system. An obvious difference between the two considerations is in the choice of the CF coordinate: Wang et al. adopt $\boldsymbol{x}^{\mathrm{c}}$ (or $\tilde{\boldsymbol{x}}$ ) as the coordinate of a CF, while we use $\boldsymbol{x}$. The results obtained in the two considerations are identical: Eq. (43) is exactly the side jump obtained by Wang et al. [15].

In the microscopic level, the presence of the Berry curvature and the internal structure of CFs in the dipole picture do introduce differences. In our theory, the impurity potential are coupled to electrons. It leads to a phase factor in the first term of Eq. (42). It is inconsequential because its contribution to the phase angle of the scattering matrix element is of the higher order in $q l_{B}$. The more fundamental difference is in Eq. (40), which is different from the usual Peierls substitution by a factor $\sim 1 / \sqrt{D}$. It is also inconsequential because it is canceled by the $D$ factor in the CF dispersion Eq. (21). The resulting perturbation Eq. (42) is identical to that of Wang's theory except for the aforementioned inconsequential difference in the effective impurity potential.

\section{Compared to Geraedts et al.'s result}

As an attempt to determine the Berry phase and Berry curvature of CFs, Geraedts et al. numerically calculate the matrix element of the density operator $\hat{\rho}_{-\boldsymbol{q}}=\sum_{a} \exp \left(\mathrm{i} \boldsymbol{q} \cdot \boldsymbol{r}_{a}\right)$ between two Rezayi-Read states with different sets of wave vectors [41]. In Ref. [44], we argue that their calculation could be regarded as a "first-principles" determination of the scattering matrix element from the microscopic wave function. It is then interesting to see how the scattering matrix element Eq. (42) determined from an effective picture is compared to the "first-principles" result.

We find that the simple formula Eq. (42) captures all essential features observed in Geraedts et al.'s calculation. To see that, we rewrite Eq. (42) in the limit $\boldsymbol{q} \rightarrow 0$ as

$$
V_{\tilde{\boldsymbol{p}}^{\prime}, \tilde{\boldsymbol{p}}} \approx-\mathrm{i} \frac{2 \tilde{V}_{\mathrm{eff}}(\boldsymbol{q})}{\hbar q} \tilde{p} \sin \theta \exp \left(\mathrm{i} \frac{\hbar q}{2 \tilde{p} \sin \theta}\right),
$$

where $\theta$ denotes the polar angle from $\tilde{\boldsymbol{p}}$ to $\boldsymbol{q}$, and $\tilde{V}_{\text {eff }}(\boldsymbol{q})$ in the current context is the effective potential induced by a singlemode scalar potential $V(\boldsymbol{r})=\exp (\mathrm{i} \boldsymbol{q} \cdot \boldsymbol{r})$. The formula predicts that (a) when $\boldsymbol{q}$ is parallel to $\tilde{\boldsymbol{p}}$, i.e., $\theta=0$, the matrix element vanishes; (b) when $\boldsymbol{q}$ is perpendicular to $\tilde{\boldsymbol{p}}$, i.e., $\theta= \pm \pi / 2$, the matrix element has a phase equal to $\pm(\hbar q / 2 \tilde{p}-\pi / 2)$; (c) traversing anticlockwise an circular path around the origin of the momentum space and cumulating the first part of the phase, one always obtains a total phase $\pi$, independent of 
the radius of the path. These are exactly what are observed in Geraedts et al.'s work.

The phase obtained from the scattering matrix element is in general not the Berry phase [44]. Geraedts et al. interpret the lack of dependence of the cumulated phase on the radius of the circular path as a manifestation of the singular distribution of the Berry curvature in a massless Dirac cone. However, we see that it is also a property of the scattering matrix element Eq. (42) even though it is for a system with a uniform Berry curvature.

Nonetheless, Geraedts et al.'s result does provide a "first principles" support for the model of impurity scattering shown in Sec. IV B.

\section{EXTRINSIC RESPONSE}

\section{A. Boltzmann equation}

In this section, we determine the extrinsic response of CFs. It is the contribution from the deviation of the non-equilibrium distribution of the system from the local equilibrium distribution Eq. (30). The non-equilibrium distribution function $f=f(t, \boldsymbol{x}, \boldsymbol{p})$ is determined by the Boltzmann equation

$$
\partial_{t} f+\dot{\boldsymbol{x}} \cdot\left(\partial_{\boldsymbol{x}} f\right)+\dot{\boldsymbol{p}} \cdot\left(\partial_{\boldsymbol{p}} f\right)=\left.\frac{\partial f}{\partial t}\right|_{\text {coll }},
$$

where $\partial f /\left.\partial t\right|_{\text {coll }}$ denotes the collision contribution of impurity scattering.

The collision contribution can be explicitly specified. In the last section, we show that CFs undergo no side jumps when scattered. It greatly simplifies our consideration because we can model the collision term as usual for elastic scattering [45]:

$$
\left.\frac{\partial f}{\partial t}\right|_{\text {coll }}=-\int\left[d \boldsymbol{p}^{\prime}\right] D W_{\boldsymbol{p}, \boldsymbol{p}^{\prime}}\left[f(t, \boldsymbol{x}, \boldsymbol{p})-f\left(t, \boldsymbol{x}, \boldsymbol{p}^{\prime}\right)\right],
$$

where $W_{\boldsymbol{p}, \boldsymbol{p}^{\prime}}$ denotes the transition probability from state $\boldsymbol{p}^{\prime}$ to $\boldsymbol{p}$. For elastic scattering, the energy conservation requires $W_{\boldsymbol{p}, \boldsymbol{p}^{\prime}} \propto \delta\left[\mathcal{E}(\boldsymbol{p})-\mathcal{E}\left(\boldsymbol{p}^{\prime}\right)\right]$.

The solution of the Boltzmann equation can be decomposed into

$$
f(t, \boldsymbol{x}, \boldsymbol{p})=f_{0}(t, \boldsymbol{x}, \boldsymbol{p})+f_{1}(t, \boldsymbol{x}, \boldsymbol{p}),
$$

where $f_{0}$ is the local equilibrium distribution specified in Eq. (30), and $f_{1}$ is the deviation induced by driving fields. It is obvious that $f_{0}$ fulfills the condition of detailed balance, i.e., the collision term vanishes for $f_{0}$.

The collision term can be further simplified. We assume that the scattering is approximately isotropic, $W_{p, p^{\prime}}=$ $W \delta\left[\mathcal{E}(\boldsymbol{p})-\mathcal{E}\left(\boldsymbol{p}^{\prime}\right)\right]$. By substituting it into the collision term, and noting that $\mathcal{E}(\boldsymbol{p})$ and $\mathcal{E}\left(\boldsymbol{p}^{\prime}\right)$ can be replaced with $\varepsilon(\boldsymbol{p})$ and $\varepsilon\left(\boldsymbol{p}^{\prime}\right)$ since $f_{1}$ has already been in the linear order of driving fields, we have [46]

$$
\left.\frac{\partial f}{\partial t}\right|_{\text {coll }}=-\frac{f_{1}-\bar{f}_{1}}{\tau},
$$

where $1 / \tau=\int\left[d \boldsymbol{p}^{\prime}\right] D W \delta\left(\varepsilon_{\boldsymbol{p}}-\varepsilon_{\boldsymbol{p}^{\prime}}\right)$ is the reciprocal of the relaxation time, and

$$
\bar{f}_{1}=\frac{1}{2 \pi} \int_{0}^{2 \pi} f_{1}(\phi) d \phi
$$

is the average of $f_{1}$ over the polar angle $\phi$ of the momentum $\boldsymbol{p}$. Strictly speaking, the transition probability for our case must be anisotropic, as evident in Eq. (42). However, in the regime $q l_{B} \ll 1$ we are interested in, the anisotropy will not introduce qualitative differences. Therefore, we ignore the anisotropy for simplicity. The generalization for anisotropic scattering is straightforward and can be found in Ref. [46].

To solve the Boltzmann equation to the linear order of driving fields, it suffices to solve for single-mode fields $[\boldsymbol{E}(t, \boldsymbol{x}), \boldsymbol{e}(t, \boldsymbol{x})]=(\boldsymbol{E}, \boldsymbol{e}) \exp (-\mathrm{i} \omega t+\mathrm{i} \boldsymbol{q} \cdot \boldsymbol{x})$. The solution has the form $f_{1}(t, \boldsymbol{x}, \boldsymbol{p})=f_{1} \exp (-\mathrm{i} \omega t+\mathrm{i} \boldsymbol{q} \cdot \boldsymbol{x})$. By substituting Eqs. (26) and (27) into Eq. (46), we have

$$
\begin{gathered}
{\left[-1 / \tau+\mathrm{i} \omega-\mathrm{i} \boldsymbol{v} \cdot \boldsymbol{q}-\omega_{\mathrm{c}}^{*}(\hat{z} \times \boldsymbol{p}) \cdot \partial_{\boldsymbol{p}}\right] f_{1}} \\
=e \boldsymbol{v} \cdot(\boldsymbol{e}+\beta \boldsymbol{E})\left(-\frac{\partial f_{0}}{\partial \varepsilon}\right)-\frac{\overline{f_{1}}}{\tau},
\end{gathered}
$$

where $\boldsymbol{v}=\boldsymbol{p} / m^{*}, \beta$ is defined in Eq. (4), and $\omega_{\mathrm{c}}^{*}=e \tilde{b} / m^{*}$ is the effective CF cyclotron frequency. The solutions of the equation for $v=1 / 2$ and $v \neq 1 / 2$ will be discussed in the following subsections.

With the distribution function, the extrinsic contribution to the $\mathrm{CF}$ (vortex) current density can be determined by

$$
\begin{gathered}
\boldsymbol{j}_{1}=-e \int[d \boldsymbol{p}] D f_{1} \dot{\boldsymbol{x}} \approx-e \int[d \boldsymbol{p}] D f_{1} \frac{\boldsymbol{p}}{m} . \\
\text { B. } \boldsymbol{v}=\mathbf{1 / 2}
\end{gathered}
$$

We first consider the case of half-filling. In this case, the effective magnetic field $\tilde{b}=0$, therefore $\omega_{\mathrm{c}}^{*}=0$. Equation (51) can be readily solved. We have

$$
f_{1}=\frac{e \boldsymbol{v} \cdot(\boldsymbol{e}+\beta \boldsymbol{E})\left(-\frac{\partial f_{0}}{\partial \varepsilon}\right)-\bar{f}_{1} / \tau}{-1 / \tau+\mathrm{i} \omega-\mathrm{i} \boldsymbol{q} \cdot \boldsymbol{v}} .
$$

By averaging both sides of Eq. (53) over the polar angle of the momentum, we obtain a self-consistent equation for determining $\bar{f}_{1}$. After solving the equation, we have

$$
\overline{f_{1}}=\tau e \overline{\boldsymbol{v}} \cdot(\boldsymbol{e}+\beta \boldsymbol{E})\left(-\frac{\partial f_{0}}{\partial \varepsilon}\right),
$$

where $\quad \overline{\boldsymbol{v}}=-i v_{\mathrm{F}} \boldsymbol{q} l /\{(1-i \omega \tau)(1+\gamma)[1-\gamma(1-i \omega \tau)]\}$, $v_{\mathrm{F}}=\hbar k_{\mathrm{F}} / m^{*}$ is the CF Fermi velocity, $l=v_{\mathrm{F}} \tau$ is the mean free path of CFs, and

$$
\gamma=\sqrt{1+\frac{(q l)^{2}}{(1-i \omega \tau)^{2}}} .
$$

The solution of the Boltzmann equation can be written as

$$
f_{1}=\frac{e(\boldsymbol{v}-\overline{\boldsymbol{v}}) \cdot(\boldsymbol{e}+\beta \boldsymbol{E})}{-1 / \tau+\mathrm{i} \omega-\mathrm{i} \boldsymbol{q} \cdot \boldsymbol{v}}\left(-\frac{\partial f_{0}}{\partial \varepsilon}\right) .
$$

Substituting Eq. (56) into Eq. (52), we obtain

$$
\begin{gathered}
\boldsymbol{j}_{1}=\tilde{\sigma}(\boldsymbol{e}+\beta \boldsymbol{E}), \\
\tilde{\sigma}_{11}=\frac{2 \sigma_{0}}{(1-\mathrm{i} \omega \tau)(1+\gamma)} \frac{\mathrm{i} \omega \tau}{1-\gamma(1-\mathrm{i} \omega \tau)}, \\
\tilde{\sigma}_{22}=\frac{2 \sigma_{0}}{(1-\mathrm{i} \omega \tau)(1+\gamma)},
\end{gathered}
$$


where $\sigma_{0}=e^{2} n \tau / m^{*}$ is the Drude conductivity, $n$ is the density of CFs, and the subscripts 1 and 2 denote the longitudinal $(\| \boldsymbol{q})$ and transverse $(\| \hat{z} \times \boldsymbol{q})$ directions, respectively. The nondiagonal elements of $\tilde{\sigma}$ vanish at half-filling.

It is important to note that the extrinsic response is not driven by the effective electric field $\tilde{\boldsymbol{e}}$ but by $\boldsymbol{e}+\beta \boldsymbol{E}$. As a result, $\boldsymbol{E}$ is not equivalent to the CS electric field $\boldsymbol{e}$ in driving the response. It manifests the fact that CFs in the dipole picture are not point particles and subject to the dipole correction from which the $\beta$ matrix arises. The dipole correction is derived from the internal structure of CFs. This is in contrast with the Dirac CF theory, in which a $\mathrm{CF}$ is regarded as a point particle and the dipole moment is introduced only as an after-thought for fulfilling the requirement of the Galilean invariance $[16,47]$.

\section{C. $v \neq 1 / 2$}

Away from half filling, the effective magnetic field $\tilde{b}=b-$ $B$ is nonzero. Solving Eq. (51) becomes more complicated. To this end, we note that Eq. (51) is identical to the Boltzmann equation for ordinary particles albeit with a driving field $\boldsymbol{e}+$ $\beta \boldsymbol{E}$. For ordinary systems, Mirlin and Wölfle obtain a solution in Ref. [46]. Their solution can be adapted to our case by noting the relation $-f_{0}^{\prime}(\varepsilon(\boldsymbol{p}))=D^{-1} \delta\left(\left(\boldsymbol{p}^{2}-p_{\mathrm{F}}^{2}\right) / 2 m^{*}\right)$. We obtain

$$
\begin{gathered}
\tilde{\sigma}_{11}=-2 \tilde{\sigma}_{0} \frac{\mathrm{i} \omega \tau}{(q l)^{2}}\left(1-\mathrm{i} \omega \tau \frac{A_{00}}{A_{00}-\omega_{\mathrm{c}}^{*} \tau}\right), \\
\tilde{\sigma}_{22}=\frac{2 \tilde{\sigma}_{0}}{\omega_{\mathrm{c}}^{*} \tau}\left(\frac{A_{0 s}^{2}}{A_{00}-\omega_{\mathrm{c}}^{*} \tau}+A_{s s}\right), \\
\tilde{\sigma}_{12}=-\tilde{\sigma}_{21}=-2 \tilde{\sigma}_{0} \frac{\omega}{v_{\mathrm{F}} q} \frac{A_{0 s}}{A_{00}-\omega_{\mathrm{c}}^{*} \tau},
\end{gathered}
$$

where $\tilde{\sigma}_{0}=\sigma_{0} / D \equiv \tilde{n} e^{2} \tau / m^{*}$ is the Drude conductivity for a system with the effective density $\tilde{n}=n / D=e B / 2 h$, and the coefficients $A_{i j}$ are defined by

$$
\begin{aligned}
A_{i j} & =\frac{1}{2 \pi} \int_{0}^{2 \pi} d \phi \psi_{i}(\phi) \int_{\mp \infty}^{\phi} d \phi^{\prime} \psi_{j}\left(\phi^{\prime}\right) \exp \left\{K\left(\phi, \phi^{\prime}\right)\right\}, \\
K\left(\phi, \phi^{\prime}\right) & =-\frac{\phi-\phi^{\prime}}{\omega_{\mathrm{c}}^{*} \tau}(1-\mathrm{i} \omega \tau)-\frac{\mathrm{i} q v_{\mathrm{F}}}{\omega_{\mathrm{c}}^{*}}\left(\sin \phi-\sin \phi^{\prime}\right), \\
\psi_{0}(\phi) & =1, \quad \psi_{s}(\phi)=\sin \phi,
\end{aligned}
$$

where the lower bound of the integral over $\phi^{\prime}$ is $-\infty(\infty)$ for $\tilde{b}>0(\tilde{b}<0)$.

The result has an important feature: it is identical to the response of a system with an effective density $\tilde{n}=e B / 2 h$ and a Fermi wave vector $k_{\mathrm{F}}=\sqrt{4 \pi \tilde{n}}=\sqrt{e B / \hbar}$, which are exactly the parameters of a Dirac CF system [16]. The effective density is set by the magnetic field instead of the electron density, exactly the swap of notions observed in the Dirac CF theory.

The formula hides a subtle difference from the HLR theory, which could have an experimental effect. At finite $q$, the formula predicts a magneto-conductivity oscillation with respect to $q v_{\mathrm{F}} /\left|\omega_{\mathrm{c}}^{*}\right|=q R_{\mathrm{c}}^{*}$, where $R_{\mathrm{c}}^{*}=\hbar k_{\mathrm{F}} / e|\tilde{b}|$ is the effective cyclotron radius of CFs $[4,8,46]$ (see Sec. VIII). Because the Fermi wave vector is now determined by the magnetic field, the magneto-conductivity oscillation will show asym- metry about $v=1 / 2$ in the positions of conductivity extrema [30], similar to that observed in recent geometric resonance experiments $[48,49]$.

\section{ELECTROMAGNETIC RESPONSE}

\section{A. CF current}

Combining the intrinsic response Eq. (33) and the extrinsic response Eq. (57) of the CF current, we have

$$
\boldsymbol{j}=-\sigma_{\mathrm{CS}}(\boldsymbol{e}+\boldsymbol{E})+\tilde{\sigma}(\boldsymbol{e}+\beta \boldsymbol{E}) .
$$

It is important to note that $\tilde{\sigma}$ is a Fermi surface property. As we have shown in the last section, it is identical to that of an effective Dirac CF system.

To determine the actual response to external EM fields, we apply the self-consistent condition Eq. (7), and find

$$
\boldsymbol{e}=\left(\tilde{\sigma}^{-1} \sigma_{\mathrm{CS}}-\beta\right) \boldsymbol{E} .
$$

Substituting the relation into Eq. (7), we obtain

$$
\boldsymbol{j}=\left(-\sigma_{\mathrm{CS}} \tilde{\sigma}^{-1} \sigma_{\mathrm{CS}}+\sigma_{\mathrm{CS}} \beta\right) \boldsymbol{E} .
$$

It yields a conductivity tensor exactly the form of Eq. (3).

\section{B. Emergence of the Dirac CF theory}

To elucidate how a Dirac CF-like EM response emerges in the dipole picture, we identify the extrinsic contribution $j_{1}=$ $\boldsymbol{j}-\boldsymbol{j}_{0}=\boldsymbol{j}+\sigma_{\mathrm{CS}} \tilde{\boldsymbol{e}}$ as the "Dirac CF" current $\tilde{\boldsymbol{j}}$, and $\boldsymbol{j}_{\mathrm{D}}=$ $\boldsymbol{j}-\sigma_{\mathrm{CS}} \boldsymbol{E}$ as the "Dirac electron" current [16]. By applying the equations of continuity for $\mathrm{CF}$ and electron densities, we can generalize them to the densities $\tilde{\rho}=\rho+\left(e^{2} / 2 h\right) \tilde{b}$ and $\rho_{\mathrm{D}}=\rho+e^{2} B / 2 h$, respectively. We thus have $j_{\mathrm{D}}^{\mu}=j^{\mu}+$ $\left(e^{2} / 2 h\right) \epsilon^{\mu \nu \gamma} \partial_{\nu} A_{\gamma}$. Substituting the CS self-consistent conditions Eqs. $(6,7)$, or $j^{\mu}=\left(e^{2} / 2 h\right) \epsilon^{\mu \nu \gamma} \partial_{\nu} a_{\gamma}$ into the relation, we obtain

$$
j_{\mathrm{D}}^{\mu}=\frac{e^{2}}{2 h} \epsilon^{\mu \nu \gamma} \partial_{\nu} \tilde{a}_{\nu}=j_{0}^{\mu} .
$$

We find that the "Dirac electron" current is nothing but the intrinsic current determined in Eq. (33). Substituting the relation and the CS self-consistent conditions into the identity $\tilde{j}=j-j_{0}$, We obtain

$$
\tilde{j}^{\mu}=-\frac{e^{2}}{2 h} \epsilon^{\mu \nu \gamma} \partial_{\nu} A_{\gamma} .
$$

The two relations Eqs. (67) and (68) are exactly the selfconsistent conditions yielded by the action of the Dirac $\mathrm{CF}$ theory [16].

We can rewrite the action Eq. (16) in terms of $\tilde{j}$ and the effective fields $\tilde{a}$, we have

$$
\begin{aligned}
S=\int d^{2} \boldsymbol{x} d t & {\left[\frac{e^{2}}{4 h} \epsilon^{\mu \nu \gamma} A_{\mu} \partial_{\nu} A_{\gamma}-\frac{e^{2}}{2 h} \epsilon^{\mu \nu \gamma} \tilde{a}_{\mu} \partial_{\nu} A_{\gamma}\right.} \\
& \left.-\tilde{a} \cdot \tilde{j}+L_{\text {Dipoles }}\right] .
\end{aligned}
$$

It has the same gauge structure as the Dirac CF action, although its particles are governed by a different Lagrangian $L_{\text {Dipoles }}=\boldsymbol{M} \cdot \boldsymbol{B}-\mathcal{E}-\mathcal{E}_{0}$ with $\mathcal{E}_{0}=\tilde{a}\left[j_{\mathrm{D}}\right] \cdot j_{\mathrm{D}} / 2$ and $\tilde{a}\left[j_{\mathrm{D}}\right]$ determined by solving Eq. (67). Nonetheless, as far as Fermisurface properties, e.g., the response of $\tilde{j}$ to external fields, 
are concerned, the particles are indistinguishable from Dirac CFs because they have the same effective density, Fermi wave vector and Berry phase (see Sec. VC). We thus find that a Dirac CF effective theory emerges from the dipole picture.

Transforming the action Eq. (16) to Eq. (69) redefines the vacuum of the effective theory from an empty (or filled) LL of electrons to a half-filled LL of vortices. It is feasible because the intrinsic response Eq. (33) contributes a half-quantized Hall conductance, which is independent of the filling factor and not altered by impurity scattering. We can interpret the intrinsic response as the contribution from the redefined vacuum, $\mathcal{E}_{0}$ as the polarization energy of the vacuum, and the remainder of the response as from an effective Dirac CF system.

\section{Electron current}

The electron current $\boldsymbol{j}^{\mathrm{e}}$ is the current actually measured in experiments. It is related to the CF current $\boldsymbol{j}$ by Eq. (12). To determine the electron current, we need to determine $\boldsymbol{P}$ and $\boldsymbol{M}$.

To determine the dipole density $\boldsymbol{P}$, we make use of Eq. (26). Applying the average $-e \int[d \boldsymbol{p}] D f$ to both sides of the equation, we obtain

$$
\boldsymbol{j}=\tilde{\omega}_{B} \hat{z} \times \boldsymbol{P}-\sigma_{\mathrm{CS}} \boldsymbol{e} .
$$

Applying the self-consistent condition Eq. (7), we find

$$
\boldsymbol{P}=0 .
$$

For the magnetization density $\boldsymbol{M}$, it is easy to see that $\boldsymbol{M}$ is proportional to $\boldsymbol{B}$ and $\boldsymbol{b}$. Since we ignore all variations of $\boldsymbol{B}$ and $\boldsymbol{b}$ for the moment, we conclude that $\boldsymbol{M}$ does not contribute to the response of $j^{\mathrm{e}}$.

We thus have

$$
j^{\mathrm{e}}=j
$$

when only the dipole correction is considered.

\section{QUADRUPOLE AND MAGNETIC CORRECTIONS}

In the previous sections, we ignore the quadrupole term in Eq. (20) as well as the temporal and spatial dependences of the magnetic fields. In this section, we will consider their effects on the EM response.

The corrections can be classified by orders of $q l_{B}$. The EM response determined in the last section is accurate only to the zeroth order of $q l_{B}$. By considering the electric quadrupole correction in Eq. (21), we can determine the response accurate to the linear order of $q l_{B}$. Because $\partial_{t} \boldsymbol{B}=-\boldsymbol{\nabla} \times \boldsymbol{E}$ is of the same order of the quadrupole correction, it should be considered on an equal footing. On the other hand, corrections due to the spatial gradient of $\boldsymbol{B}$ is of the quadratic order in $q l_{B}$, and considering them requires a multipole expansion of $j^{\mathrm{e}}$ to at least the order of the magnetic quadrupole [38]. It is beyond the scope of the current study.

Corrections due to the variation of $\boldsymbol{b}$ should be fully accounted for. Even thought $\boldsymbol{b}$ looks like the counterpart of $\boldsymbol{B}$, it is actually equivalent to the density because of the selfconsistent condition Eq. (6). Since it is not subject to the approximation of the multipole expansions, considering its gradients will not introduce inconsistencies. As we will see, its corrections are essential for obtaining a density response function of $\mathrm{CFs}$ with a correct static limit.

For simplicity, we consider these corrections only for half filling.

\section{A. Quadrupole correction}

By using Eq. (19) and the complete form of Eq. (20), we determine modifications to the equations of motion Eqs. (22) and (23) due to the quadrupole correction to the energy and the time dependence of $B$. We have

$$
\begin{gathered}
\dot{\boldsymbol{x}}=\frac{b}{B} \frac{\boldsymbol{p}}{m^{*}}-\frac{1}{e B} \dot{\boldsymbol{p}} \times \hat{z}+\frac{1}{e B^{2}} \hat{z} \times \nabla \boldsymbol{E} \cdot(\boldsymbol{p} \times \hat{z}), \\
\dot{\boldsymbol{p}}=-\frac{1}{B} \nabla \boldsymbol{E} \cdot(\boldsymbol{p} \times \hat{z}),
\end{gathered}
$$

where we keep only terms related to $\boldsymbol{\nabla} \boldsymbol{E}$ since only they are relevant to the quadrupole correction for the linear response to EM fields. Substituting Eq. (74) into Eq. (73), we obtain $\dot{\boldsymbol{x}}=$ $\boldsymbol{p} / m^{*}$, i.e., the quadrupole correction does not change Eq. (26).

The Boltzmann equation can then be solved. We decompose the distribution function into $f=f_{0}+f_{1}$, where $f_{0}$ still assumes the same form as Eq. (30) but with the quadrupole term in Eq. (20) included. It is easy to see that it introduces no correction to $\boldsymbol{j}_{0}$. The correction to $f_{1}$ is

$$
\Delta f_{1}=\frac{e\left(\boldsymbol{v}^{\prime}-\bar{v}^{\prime}\right) \cdot \boldsymbol{E}}{-1 / \tau+\mathrm{i} \omega-\mathrm{i} \boldsymbol{q} \cdot \boldsymbol{v}}\left(-\frac{\partial f_{0}}{\partial \varepsilon}\right),
$$

where $\boldsymbol{v}^{\prime}=-\left(\omega / 2 \tilde{\omega}_{B}^{2}\right) \boldsymbol{q} \cdot(\hat{z} \times \boldsymbol{v})(\hat{z} \times \boldsymbol{v})$ and $\overline{\boldsymbol{v}}^{\prime}=-v_{\mathrm{F}}\left(\boldsymbol{q} l_{B}\right)$ $\left(\omega / \tilde{\omega}_{B}\right) \gamma /[2(1+\gamma)(1-\gamma(1-i \omega \tau))]$.

The quadrupole correction to $\boldsymbol{j}_{1}$ can then be determined by using Eq. (52). It is obvious from Eq. (20) that the quadrupole term has a similar effect as the dipole term in making $\boldsymbol{E}$ nonequivalent to $\boldsymbol{e}$. Therefore, the quadrupole correction can be characterized by a correction to the $\beta$ matrix. We have

$$
\begin{aligned}
& \Delta \beta_{11}=-\frac{\left(q l_{B}\right)^{2}}{4} \frac{1+\gamma(1-i \omega \tau)}{(1+\gamma)(1-i \omega \tau)} \approx 0, \\
& \Delta \beta_{22}=-\frac{q l_{B}}{4} \frac{i \omega(q l)}{\tilde{\omega}_{B}} \frac{1}{(1+\gamma)(1-i \omega \tau)},
\end{aligned}
$$

and nondiagonal elements vanish. The correction then propagates to Eq. (66) for the total EM response.

To determine the electron current $\boldsymbol{j}^{\mathrm{e}}$, we need to determine the displacement current $\boldsymbol{J}^{\mathrm{Q}}=-\partial \boldsymbol{\nabla} \cdot \mathbb{Q} / \partial t$. Note that $\boldsymbol{P}=0$ is still true since Eq. (26) is not modified by the quadrupole correction. One may evaluate $\boldsymbol{J}^{\mathrm{Q}}$ directly, or apply the Onsager relation, which yields in the current context

$$
\boldsymbol{J}^{\mathrm{Q}}=(\Delta \beta \tilde{\sigma})(\boldsymbol{e}+\beta \boldsymbol{E})=\Delta \beta \sigma_{\mathrm{CS}} \boldsymbol{E},
$$

where we apply Eq. (65) to get the last form. The total correction to the electron conductivity tensor of the system is

$$
\Delta \sigma=\sigma_{\mathrm{CS}} \Delta \beta+\Delta \beta \sigma_{\mathrm{CS}},
$$

where the first term is from the $\Delta \beta$ correction to Eq. (66). To the linear order of $q l_{B}$, it contributes a correction to the Hall conductivity

$$
\Delta \sigma_{x y}=-\frac{e^{2}}{h} \frac{q l_{B}}{8} \frac{\mathrm{i} \omega}{\tilde{\omega}_{B}} \frac{q l}{(1+\gamma)(1-i \omega \tau)} .
$$




\section{B. Magnetic correction}

In this subsection, we determine corrections due to the variation of the CS magnetic field $\delta b$. It has two effects. First, it modifies the equations of motion, and introduces corrections to the responses of the CF current. Second, it gives rise to a magnetization current because the $\mathrm{CF}$ dispersion Eq. (21) implies that each $\mathrm{CF}$ carries a magnetic moment $\boldsymbol{m}_{b}=-\left(p^{2} / 2 m^{*} B\right) \hat{z}$ with respect to the CS magnetic field. Both the effects should be considered.

First, we determine the corrections to the $\mathrm{CF}$ responses. In this case, the local equilibrium distribution is assumed to be

$$
f_{0}(\boldsymbol{x}, \boldsymbol{p}, t)=n_{\mathrm{F}}\left(\varepsilon(\boldsymbol{p})-\varepsilon\left(\boldsymbol{p}_{\mathrm{F}}\right)\right),
$$

where we drop terms in the energy irrelevant to $\delta b$ and assume a local chemical potential $\mu(\boldsymbol{x})=\varepsilon\left(\boldsymbol{p}_{\mathrm{F}}\right)=b(\boldsymbol{x}) p_{\mathrm{F}}^{2} / 2 m^{*} B$ so that the distribution has a uniform density. Note that the choice of $\mu(\boldsymbol{x})$ does not affect the steady-state solution of the Boltzmann equation.

We can then determine the correction to the intrinsic current $\boldsymbol{j}_{0}$. $\delta b$ introduces extra forces $-\left(p^{2} / 2 m^{*} B\right) \nabla \delta b-$ $e \delta b \dot{\boldsymbol{x}} \times \hat{z}$ into the system. As a result, Eqs. (26) and (27) are modified to

$$
\begin{gathered}
\dot{\boldsymbol{x}}=\frac{\boldsymbol{p}}{m^{*}}-\frac{1}{b} \hat{z} \times \boldsymbol{e}+\frac{1}{e b} \boldsymbol{m}_{b} \times \nabla \delta b, \\
\dot{\boldsymbol{p}}=e \delta b \hat{z} \times \frac{\boldsymbol{p}}{m^{*}}-e\left(\frac{\boldsymbol{e}}{D}+\boldsymbol{E}\right)-\frac{\nabla \delta b}{b} \frac{p^{2}}{2 m^{*}} .
\end{gathered}
$$

The correction to $\boldsymbol{j}_{0}$ is

$$
\Delta \boldsymbol{j}_{0}=-\int[d \boldsymbol{p}] D f_{0} \frac{\boldsymbol{m}_{b} \times \nabla \delta b}{b}=\frac{e^{2}}{16 \pi m^{*}} \hat{z} \times \nabla \delta b .
$$

The correction to the extrinsic current $\boldsymbol{j}_{1}$ can also be determined. It is straightforward to determine that $\delta b$ introduces a correction to $f_{1}$ :

$$
\Delta f_{1}=\frac{e(\boldsymbol{v}-\overline{\boldsymbol{v}}) \cdot\left(\frac{\hbar}{2 m^{*}} \nabla \delta b\right)}{-1 / \tau+\mathrm{i} \omega-\mathrm{i} \boldsymbol{q} \cdot \boldsymbol{v}}\left(-\frac{\partial f_{0}}{\partial \varepsilon}\right),
$$

i.e., the effect of $\delta b$ is equivalent to an effective electric field $(\hbar / 2 m) \nabla \delta b$. Therefore, the correction to $\boldsymbol{j}_{1}$ is

$$
\Delta \boldsymbol{j}_{1}=\tilde{\sigma} \frac{\hbar}{2 m^{*}} \nabla \delta b .
$$

Next, we determine the magnetization current. The CS magnetization is determined by $\boldsymbol{M}_{b}=\int[d \boldsymbol{p}] D f \boldsymbol{m}_{b}$. We decompose $\boldsymbol{m}_{b}$ as $\boldsymbol{m}_{b}\left(p_{\mathrm{F}}\right)+\left[\boldsymbol{m}_{b}(p)-\boldsymbol{m}_{b}\left(p_{\mathrm{F}}\right)\right]$, and note that $f_{1}$ does not contribute to the expectation value of the second term. We obtain

$$
\boldsymbol{M}_{b}=\frac{\hbar \rho}{2 m^{*}} \hat{z}-\int[d \boldsymbol{p}] D f_{0} \frac{p^{2}-p_{\mathrm{F}}^{2}}{2 m^{*} B} \hat{z}=\frac{\hbar \rho}{4 m^{*}} \hat{z} .
$$

After applying Eq. (6), we find that the magnetization current is

$$
j^{\mathrm{m}}=\nabla \times \boldsymbol{M}_{b}=\frac{e^{2}}{16 \pi m^{*}} \hat{z} \times \nabla \delta b=\Delta \boldsymbol{j}_{0} .
$$

Summing all the corrections, we find that Eq. (64) is modified by substituting $\boldsymbol{e}$ with $\boldsymbol{e}+\left(\hbar / 2 m^{*}\right) \nabla \delta b$. After applying the self-consistent conditions Eqs. (6) and (7), we obtain

$$
\boldsymbol{j}=-\sigma_{\mathrm{CS}} \tilde{\sigma}^{-1} \sigma_{\mathrm{CS}} \boldsymbol{E}^{*}+\sigma_{\mathrm{CS}}\left[\beta \boldsymbol{E}+\frac{1}{e^{2} \tilde{\chi}_{0}} \nabla \rho\right] \text {, }
$$

where $\tilde{\chi}_{0}=-m^{*} / 2 \pi \hbar^{2}$ is the static density susceptibility of a free $\mathrm{CF}$ system at $\boldsymbol{q}=0$, and

$$
\boldsymbol{E}^{*}=\boldsymbol{E}+\frac{1}{e^{2} \tilde{\chi}_{0}} \nabla \rho .
$$

Finally, we determine the electron current $j^{\mathrm{e}}$. We need to determine the polarization density $\boldsymbol{P}$ and the magnetization density $\boldsymbol{M}$. The polarization density does not vanish due to the magnetic correction in Eq. (82). Applying the average $-e \int[d \boldsymbol{p}] D f$ to both sides of Eq. (82) and noting $-e \int[d \boldsymbol{p}] D \dot{\boldsymbol{x}} f=\boldsymbol{j}-\boldsymbol{j}^{\mathrm{m}}$ in the current context, we obtain

$$
\boldsymbol{P}=-\frac{1}{\tilde{\omega}_{B}} \frac{e^{2}}{8 \pi m^{*}} \nabla \delta b .
$$

Next, we determine the magnetization density $\boldsymbol{M}$. Each CF carries a magnetic moment with respect to $B: \boldsymbol{m}_{B}=$ $\partial L / \partial \boldsymbol{B}=(\boldsymbol{p} \times \dot{\boldsymbol{p}}) / 2 e B^{2}-\hat{z}\left(b / B^{2}\right)\left(p^{2} / 2 m^{*}\right)+\boldsymbol{m}_{B}^{\prime}$, where $\boldsymbol{m}_{B}^{\prime}$ denotes contributions from such as the $B$ dependence of the effective mass or the zero-point energy of a LL as discussed in the $\mathrm{M}^{2} \mathrm{RPA}$ theory $[13,50]$. Substituting Eq. (83), we obtain $\boldsymbol{m}_{B}=\boldsymbol{m}_{\boldsymbol{b}}+\boldsymbol{m}_{B}^{\prime}$. The terms related to driving fields in Eq. (83) are ignored because their contributions are of higher orders of $q l_{B}$.

The total electron current can then be obtained by applying Eq. (12). We note that $\boldsymbol{j}^{\mathrm{m}}$ should be subtracted from $\boldsymbol{j}$ when applying the relation. It cancels part of the contribution from $\boldsymbol{m}_{B}$. The net magnetization correction to the electron current is thus contributed only by $\boldsymbol{M}^{\prime} \equiv \int[d \boldsymbol{p}] D \boldsymbol{m}_{B}^{\prime} f$. We have

$$
\boldsymbol{j}^{\mathrm{e}}=\left(-\sigma_{\mathrm{CS}} \tilde{\sigma}^{-1} \sigma_{\mathrm{CS}}+\sigma_{\mathrm{CS}} \beta\right) \boldsymbol{E}^{*}+\nabla \times \boldsymbol{M}^{\prime} .
$$

Applying the relation $\rho(\omega, \boldsymbol{q}) \approx \rho^{\mathrm{e}}(\omega, \boldsymbol{q})=\boldsymbol{q} \cdot \boldsymbol{j}^{\mathrm{e}}(\omega, \boldsymbol{q}) / \omega$, we can rewrite the electron current as $\boldsymbol{j}^{\mathrm{e}}=\sigma \boldsymbol{E}+\nabla \times \boldsymbol{M}^{\prime}$ with the conductivity tensor $\sigma$ determined by

$$
\sigma^{-1}=\left(\sigma^{*}\right)^{-1}-\frac{\mathrm{i} q^{2}}{\omega} \frac{1}{e^{2} \tilde{\chi}_{0}}\left(\begin{array}{ll}
1 & 0 \\
0 & 0
\end{array}\right),
$$

where $\sigma^{*}=-\sigma_{\mathrm{CS}} \tilde{\sigma}^{-1} \sigma_{\mathrm{CS}}+\sigma_{\mathrm{CS}} \beta$ is the conductivity tensor with respect to $\boldsymbol{E}^{*}$.

\section{Density response function}

From Eq. (93), we can determine the density response function $\chi$ of electrons by using the relation $\chi(\omega, \boldsymbol{q})=$ $-\mathrm{i} q^{2} \sigma_{11}(\omega, \boldsymbol{q}) / e^{2} \omega$. We have

$$
\frac{1}{\chi}=\frac{1}{\tilde{\chi}_{0}}+\frac{\mathrm{i} \omega \tilde{\sigma}_{22}}{q^{2}} \frac{(2 h / e)^{2}}{1+\frac{2 h}{e^{2}} \frac{\mathrm{i} \omega}{\tilde{\omega}_{B}} \tilde{\sigma}_{22}},
$$

where $\tilde{\sigma}_{22}$ is the transverse component of $\tilde{\sigma}$ shown in Eq. (59).

It can be shown that $\chi$ is related to the magnetic susceptibility of CFs. The first term in the right hand side of 
Eq. (94), which is contributed by the magnetic correction, can be absorbed into the second term as a correction to $\tilde{\sigma}_{22}$. In the low-frequency limit $\omega \rightarrow 0$, we have $\Delta \tilde{\sigma}_{22} \approx$ $(e / 2 h)^{2} q^{2} / \mathrm{i} \omega \tilde{\chi}_{0}$. Because the magnetic susceptibility of CFs is related to the transverse component of the CF conductivity tensor by $\tilde{\chi}^{\mathrm{m}}=\mathrm{i} \omega\left[\tilde{\sigma}_{22}+\Delta \tilde{\sigma}_{22}\right] / q^{2}[51]$, we have

$$
\frac{1}{\chi_{0}}=\left(\frac{2 h}{e}\right)^{2} \tilde{\chi}_{0}^{\mathrm{m}},
$$

where $\chi_{0}\left(\tilde{\chi}_{0}^{\mathrm{m}}\right)$ denotes the static density (magnetic) susceptibility of electrons (CFs).

Equation (95) implies a self-consistency condition for the model of CFs. In Sec. IV B, we assume the relation $\delta b(\boldsymbol{x})=-\left(2 m^{*} / e \hbar\right) V_{\mathrm{eff}}(\boldsymbol{x})$ for determining the magnitude of the fluctuating CS magnetic field. It leads to the conclusion of no-side-jumps in Sec. IV B, and our derivation of the extrinsic response is based on the conclusion. The relation requires that the density response function of electrons should have the long-wavelength static limit $\chi_{0}=\tilde{\chi}_{0}$. Therefore, to make our considerations self-consistent, the static density susceptibility $\tilde{\chi}_{0}$ of CFs should be related to $\tilde{\chi}_{0}^{\mathrm{m}}$ by the same relation as Eq. (95). It is easy to verify that the relation indeed holds for our semiclassical model. However, our semiclassical approach cannot fully determine the magnetic susceptibility without taking account of quantum effects (e.g., Landau diamagnetism) and specifying an effective hamiltonian to the quadratic order of $\delta b$ [52]. Therefore, we need to assume that a full effective hamiltonian of CFs has these corrections canceled.

\section{AN EXPERIMENTAL TEST}

The longitudinal conductivity $\sigma_{11}(\omega, \boldsymbol{q})$ of a twodimensional electron gas (2DEG) at finite $\boldsymbol{q}$ can be measured by using a surface acoustic wave (SAW) that traverses the 2DEG. The SAW is attenuated by the $2 \mathrm{DEG}$ and its velocity is altered [53-55]. It is shown that the shift of the sound velocity $v_{\mathrm{s}}$ is related to $\sigma_{11}(\omega, \boldsymbol{q})$ by $[46,53,56]$

$$
\frac{\Delta v_{\mathrm{s}}}{v_{\mathrm{s}}}=\frac{\alpha^{2}}{2} \operatorname{Re} \frac{1}{1+\mathrm{i} \sigma_{11}(\omega, \boldsymbol{q}) / \sigma_{\mathrm{m}}},
$$

where $\alpha^{2} / 2=3.2 \times 10^{-4}$ is the piezoelectric coupling constant for GaAs, $\sigma_{\mathrm{m}}=2 \epsilon_{\mathrm{eff}} v_{\mathrm{s}}$, and $\epsilon_{\mathrm{eff}}$ is the effective dielectric constant for the $2 \mathrm{DEG}$.

The dipole theory (as well as the Dirac CF theory) predicts a longitudinal conductivity $\sigma_{11}(\omega, \boldsymbol{q})$ different from that of the HLR theory. The most important difference is contributed by the off-diagonal component of the $\beta$ matrix in Eq. (3), which originates from the dipole correction (see Sec. VI A). We have

$$
\sigma_{11}^{\text {Dipole }}-\sigma_{11}^{\mathrm{HLR}} \approx \mathrm{i} \frac{e^{2}}{2 h} \frac{\omega}{\tilde{\omega}_{\mathrm{B}}} .
$$

The magnitude of the correction $\left(\sim 3 \times 10^{-7} \mathrm{~S}\right.$ for the experiment considered below) turns out to be comparable to that of $\sigma_{\mathrm{m}}$. Since the correction to the imaginary part of the conductivity scales the velocity shift: $\Delta v_{\mathrm{s}} / v_{\mathrm{s}} \approx$ $\left(\alpha^{2} / 2\right)\left[1-\operatorname{Im}\left(\sigma_{11} / \sigma_{\mathrm{m}}\right)\right] /\left[\operatorname{Re}\left(\sigma_{11} / \sigma_{\mathrm{m}}\right)\right]^{2}$, there is a good chance to detect its effect in a SAW experiment.

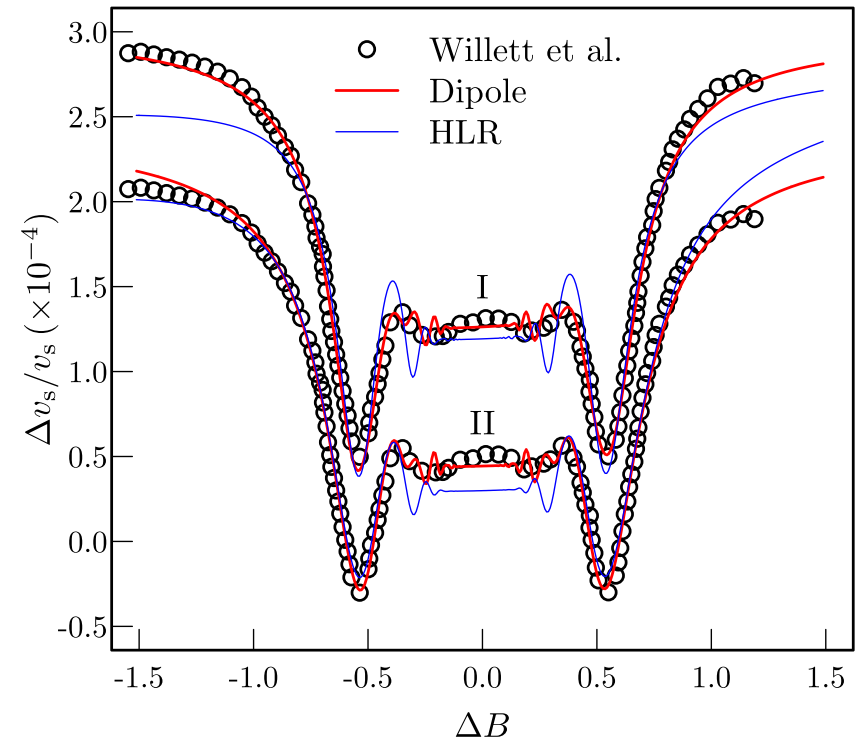

FIG. 2. Experimental data of the SAW velocity shift adapted from Ref. [57] (black circles) and fits to the data by using the dipole theory (red-thick lines) and the HLR theory (blue-thin lines). Two different ways of fittings are shown: (I) fits the data as it is by treating $\sigma_{\mathrm{m}}$ as a fitting parameter; (II) fits the data after subtracting a constant background and fixes the value of $\sigma_{\mathrm{m}}$ to $6.8 \times 10^{-4} \mathrm{~S}$. For clarity, (II) is offset vertically by $-0.8 \times 10^{-4}$.

As a test of our theory and an effort of looking for the dipole correction, we fit the data presented in Ref. [57]. The experiment is one of a series of SAW experiments carried out by Willett et al. on CF systems [6,55,57,58]. It employs a high SAW frequency $(10.7 \mathrm{GHz})$ and thus has the best data quality. The data is shown in Fig. 2.

We first fit the data as it is. We find that the dipole theory fits the data rather well. It reproduces the main features of the resonances and coincides well with the data in the whole range of $\Delta B$, as evident in Fig. 2 (denoted as I). The parameters for the fit are shown in Table I. All parameters except $\sigma_{\mathrm{m}}$ are close to their respective reference values. $\sigma_{\mathrm{m}}$, on the other hand, is approximately a factor of two larger than the expected value. In comparison, the HLR theory fits the data less well. It cannot fit the data near $\Delta B \sim 0$ and at the two ends simultaneously, and produces subresonance features that are too pronounced. Moreover, we cannot treat $m^{*}$ as a fitting parameter for the fitting to the HLR theory because it converges to an unphysical

TABLE I. The values of fitting parameters. Underlined numbers indicate values fixed in fittings. $\left(\Delta v_{\mathrm{s}} / v_{\mathrm{s}}\right)_{0}$ is the value of the back-

\begin{tabular}{|c|c|c|c|c|c|c|c|}
\hline & & $\begin{array}{c}v_{\mathrm{s}} \\
\left(10^{3} \mathrm{~m} / \mathrm{s}\right)\end{array}$ & $\begin{array}{c}m^{*} \\
\left(m_{\mathrm{e}}\right)\end{array}$ & $\begin{array}{c}\tau \\
(\mathrm{ps})\end{array}$ & $\begin{array}{c}\sigma_{\mathrm{m}} \\
\left(10^{-7} \mathrm{~S}\right)\end{array}$ & $\begin{array}{c}\alpha^{2} / 2 \\
\left(10^{-4}\right)\end{array}$ & $\begin{array}{c}\left(\Delta v_{\mathrm{s}} / v_{\mathrm{s}}\right)_{0} \\
\left(10^{-4}\right)\end{array}$ \\
\hline \multirow[t]{2}{*}{ I } & Dipole & 3.1 & 0.6 & 14 & 14 & $\underline{3.2}$ & $\underline{0}$ \\
\hline & HLR & 3.0 & $\underline{0.3}$ & 7.3 & 12 & 3.2 & $\overline{\overline{0}}$ \\
\hline \multirow[t]{2}{*}{ II } & Dipole & 3.1 & $\overline{0.7}$ & 20 & 6.8 & $\overline{2.8}$ & $0 . \overline{8} 9$ \\
\hline & HLR & 3.1 & 0.3 & 5.7 & $\overline{6.8}$ & 4.4 & 0.30 \\
\hline & eference & 3.0 [59] & $0.8[57]$ & & $\overline{6.8}$ & 3.2 & \\
\hline
\end{tabular}
ground subtracted from the data. The last line shows the reference values of the parameters as well as their sources. 
negative value. We therefore set $m^{*}=0.3 m_{\mathrm{e}}$. Smaller values of $m^{*}$ yield slightly better fits but not much different from the one shown. The fitted value of $\sigma_{\mathrm{m}}$ is also significantly larger than its expected value.

The disparity between the fitted values of $\sigma_{\mathrm{m}}$ and its expected value is an issue. In literatures, the value of $\sigma_{\mathrm{m}}$ is usually quoted as $3.5 \times 10^{-7} \mathrm{~S}[6,55,57,58]$. The value is obtained by assuming $\epsilon_{\mathrm{eff}}=\left(\epsilon_{0}+\epsilon\right) / 2$, where $\epsilon_{0}$ and $\epsilon$ are the dielectric constants of vacuum and GaAs, respectively. The assumption is appropriate only when the 2DEG is on top of a sample surface, while in reality 2DEG is always a finite depth $d$ underneath the surface. When $q d \gtrsim 1$, which is likely true for the current case, one should use $\epsilon_{\text {eff }} \approx \epsilon$ [59]. It yields the reference value $\sigma_{\mathrm{m}}=6.8 \times 10^{-7} \mathrm{~S}$ shown in Table I. Unfortunately, it is not easy to account for the remaining disparity in the value of $\sigma_{\mathrm{m}}$. We are not aware of another mechanism that could increase $\sigma_{\mathrm{m}}$.

We therefore try to fit the data by insisting $\sigma_{\mathrm{m}}=6.8 \times$ $10^{-7} \Omega^{-1}$. In this case, both the theories cannot fit the data as they show sizable deviations and too strong resonances. Actually, in the regime $q l \gg 1(q l \approx 8$ in the current case) necessary for the observation of strong resonances, we have

$$
\sigma_{11}(\Delta B=0) \approx \frac{e^{2}}{4 h}\left(\frac{q}{k_{\mathrm{F}}} \pm \mathrm{i} \frac{\omega}{\tilde{\omega}_{\mathrm{B}}}\right)
$$

where the + and - signs are for the dipole theory and the HLR theory, respectively. The real (and dominant) part of the conductivity is set only by external parameters (the wavenumber and the density). It predicts $\Delta v_{\mathrm{s}} / v_{\mathrm{s}} \sim 0.4 \times 10^{-4}$ at $\Delta B=0$ for $\sigma_{\mathrm{m}}=6.8 \times 10^{-7} \mathrm{~S}$, much lower than the value actually observed in the experiment. Moreover, because $\sigma_{11}$ is determined by the transverse component of the CF conductivity $\tilde{\sigma}_{22}$ whose real part is not renormalized by interaction in the clean limit [60], the disparity cannot be eliminated by considering a residual interaction between $\mathrm{CFs}$.

We thus conjecture that there may exist extrinsic mechanisms, which contribute a background to the experimentally measured SAW velocity shifts. We treat the value of the background as a fitting parameter. We also treat the piezoelectric coupling constant $\alpha^{2} / 2$ as a fitting parameter because it is shown to be a function of the depth $d$ of the 2DEG layer [59]. The fittings are shown in Fig. 2 with parameters shown in Table I (denoted as II). It is evident that the dipole theory has the better fit. It yields parameters close to their respective reference values or in the reasonable regime. On the contrary, in the fitting to the HLR theory, we find sizable deviations and a fitted value of $\alpha^{2} / 2$, which is too large [61].

Although the dipole theory consistently provides better fits to the experimental data, the support to it cannot be regarded conclusive before either the value of $\sigma_{\mathrm{m}}$ or the conjectured background can be clarified. We also apply the fitting to the set of data presented in Ref. [58]. We find that both the theories can reproduce main features but with sizable quantitative deviations. More experiments may be needed for clarifying various factors that may affect the measurement. Our analysis, nevertheless, does show that SAW experiments have the potential for measuring the dipole correction and differentiating different theories of CFs.
Finally, we note that the magnetic correction shown in Eq. (93) only slightly modifies $\sigma_{11}$, and the quadrupole correction, which only alters the Hall conductivity, does not have an effect to the velocity shift. Therefore, SAW experiments can not distinguish the dipole theory from the Dirac CF theory.

\section{SUMMARY AND DISCUSSION}

In summary, we determine the EM response of CFs of the dipole picture. We show how the Dirac CF-like EM response emerges in the dipole picture (Sec. VIB). We show that a CF system has an intrinsic Hall response, which is independent of the filling factor (Sec. III A) and not altered by impurity scattering (Sec. IV). When one ascribes the intrinsic response to a redefined vacuum, the remainder of the EM response can be interpreted as from a Dirac CF system as far as the the low energy and long wavelength response is concerned.

When we go beyond the zeroth order in $q l_{B}$, deviations from the Dirac CF theory start to show up (Sec. VII). We see that the quadrupole correction gives rise to a correction to the Hall conductivity, and the magnetic correction modifies the density response function. Although it could be hard to detect these corrections in experiments, they do manifest an important fact of the dipole picture, i.e., a CF has an internal structure.

It is natural to ask whether or not the internal structure of a $\mathrm{CF}$ can be probed in experiments. In the dipole picture, a CF has a length scale $l_{B}$, comparable to its Fermi wavelength. It suggests that carefully designed experiments that can measure the Fermi wavelength could potentially probe the internal structure as well. To this end, we fit the SAW measurement data by Willett et al., and show that the contribution from the dipole correction is sizable and improves fittings. To distinguish the dipole model and the Dirac CF theory, on the other hand, we need to detect quadrupoles or higher order corrections. In Ref. [30], we propose such an experiment. We show that a geometrical resonance experiment with a modulated magnetic field will give rise to asymmetry opposite to that observed in a similar experiment but with a modulated scalar potential (or the CS magnetic field).

Our theory suggests a couple of constraints on the effective model describing the dipole, including the density-of-state factor appeared in the dispersion Eq. (21) and the relation Eq. (95) between the magnetic and density susceptibilities. In principle, one could infer an effective Hamiltonian directly from microscopic wave-functions. It is necessary to develop a scheme to do that and test the viability of our assumptions. This will be left as a future investigation.

We do not consider the effects of residual interaction between CFs for a reason. For both the HLR theory and the Dirac CF theory, the consideration is essential because both the theories rely on renormalizations to eliminate the unwelcome presences of bare parameters, i.e., the band mass for the HLR theory and the velocity of the massless Dirac cone for the Dirac CF theory. For our theory, on the other hand, the consideration is less important because our model is inferred directly from a microscopic CF wave function defined in the projected Hilbert space of a LL. Spurious dependencies on bare parameters have been eliminated from 
the beginning. The residual interaction between CFs, which should also be derived directly from the microscopic $\mathrm{CF}$ wave function, would renormalize model parameters. In contrast to the HLR theory and the Dirac CF theory, the renormalization in our theory needs not relate the parameters to their bare counterparts.

\section{ACKNOWLEDGMENTS}

This work is supported by the National Basic Research Program of China (973 Program) Grants No. 2018YFA0305603 and No. 2015CB921101 and the National Science Foundation of China Grant No. 11325416.
[1] D. C. Tsui, H. L. Stormer, and A. C. Gossard, Two-Dimensional Magnetotransport in the Extreme Quantum Limit, Phys. Rev. Lett. 48, 1559 (1982).

[2] J. Jain, Composite Fermions (Cambridge University Press, Cambridge, 2007).

[3] J. K. Jain and P. W. Anderson, Beyond the Fermi liquid paradigm: Hidden Fermi liquids, Proc. Natl. Acad. Sci. USA 106, 9131 (2009).

[4] R. Willet, Composite fermions-Experimnental findings, in Composite Fermions, edited by O. Heinonen (World Scientific, Singapore, 1998), p. 349.

[5] J. H. Smet, Ballistic transport of composite fermions in semiconductor nanostructures, in Composite Fermions, edited by $\mathrm{O}$. Heinonen (World Scientific, Singapore, 1998), p. 443.

[6] R. L. Willett, K. W. West, and L. N. Pfeiffer, Geometric Resonance of Composite Fermion Cyclotron Orbits with a Fictitious Magnetic Field Modulation, Phys. Rev. Lett. 83, 2624 (1999).

[7] J. H. Smet, S. Jobst, K. von Klitzing, D. Weiss, W. Wegscheider, and V. Umansky, Commensurate Composite Fermions in Weak Periodic Electrostatic Potentials: Direct Evidence of a Periodic Effective Magnetic Field, Phys. Rev. Lett. 83, 2620 (1999).

[8] B. I. Halperin, P. A. Lee, and N. Read, Theory of the half-filled Landau level, Phys. Rev. B 47, 7312 (1993).

[9] S. C. Zhang, T. H. Hansson, and S. Kivelson, EffectiveField-Theory Model for the Fractional Quantum Hall Effect, Phys. Rev. Lett. 62, 82 (1989).

[10] A. Lopez and E. Fradkin, Fractional quantum Hall effect and Chern-Simons gauge theories, Phys. Rev. B 44, 5246 (1991).

[11] V. Kalmeyer and S.-C. Zhang, Metallic phase of the quantum Hall system at even-denominator filling fractions, Phys. Rev. B 46, 9889 (1992).

[12] S. H. Simon and B. I. Halperin, Finite-wave-vector electromagnetic response of fractional quantized Hall states, Phys. Rev. B 48, 17368 (1993).

[13] S. H. Simon, The Chern-Simons Fermi liquid description of fractional quantum Hall states, in Composite Fermions, edited by O. Heinonen (World Scientific, Singapore, 1998), p. 91.

[14] S. A. Kivelson, D.-H. Lee, Y. Krotov, and J. Gan, Compositefermion Hall conductance at $v=1 / 2$, Phys. Rev. B 55, 15552 (1997).

[15] C. Wang, N. R. Cooper, B. I. Halperin, and A. Stern, ParticleHole Symmetry in the Fermion-Chern-Simons and Dirac Descriptions of a Half-Filled Landau Level, Phys. Rev. X 7, 031029 (2017).

[16] D. T. Son, Is the Composite Fermion a Dirac Particle?, Phys. Rev. X 5, 031027 (2015).

[17] S. D. Geraedts, M. P. Zaletel, R. S. K. Mong, M. A. Metlitski, A. Vishwanath, and O. I. Motrunich, The half-filled Landau level: The case for Dirac composite fermions, Science 352, 197 (2016).
[18] W. Pan, W. Kang, K. W. Baldwin, K. W. West, L. N. Pfeiffer, and D. C. Tsui, Berry phase and anomalous transport of the composite fermions at the half-filled Landau level, Nat. Phys. 13, 1168 (2017).

[19] D. F. Mross, J. Alicea, and O. I. Motrunich, Explicit Derivation of Duality between a Free Dirac Cone and Quantum Electrodynamics in (2+1) Dimensions, Phys. Rev. Lett. 117, 016802 (2016).

[20] M. Levin and D. T. Son, Particle-hole symmetry and electromagnetic response of a half-filled Landau level, Phys. Rev. B 95, 125120 (2017).

[21] N. Read, Theory of the half-filled Landau level, Semicond. Sci. Technol. 9, 1859 (1994).

[22] E. Rezayi and N. Read, Fermi-Liquid-Like State in a HalfFilled Landau Level, Phys. Rev. Lett. 72, 900 (1994).

[23] A. C. Balram and J. K. Jain, Nature of composite fermions and the role of particle-hole symmetry: A microscopic account, Phys. Rev. B 93, 235152 (2016).

[24] R. Shankar and G. Murthy, Towards a Field Theory of Fractional Quantum Hall States, Phys. Rev. Lett. 79, 4437 (1997).

[25] R. Shankar, Hamiltonian Description of Composite Fermions: Aftermath, Phys. Rev. Lett. 83, 2382 (1999).

[26] G. Murthy and R. Shankar, Hamiltonian theories of the fractional quantum Hall effect, Rev. Mod. Phys. 75, 1101 (2003).

[27] J. Shi and W. Ji, Dynamics of the Wigner crystal of composite particles, Phys. Rev. B 97, 125133 (2018).

[28] G. Sundaram and Q. Niu, Wave-packet dynamics in slowly perturbed crystals: Gradient corrections and Berry-phase effects, Phys. Rev. B 59, 14915 (1999).

[29] D. Xiao, M.-C. Chang, and Q. Niu, Berry phase effects on electronic properties, Rev. Mod. Phys. 82, 1959 (2010).

[30] G. Ji and J. Shi, Asymmetry of the geometrical resonances of composite fermions, Phys. Rev. B 101, 235301 (2020).

[31] D.-H. Lee, Neutral Fermions at Filling Factor $v=1 / 2$, Phys. Rev. Lett. 80, 4745 (1998).

[32] D.-H. Lee, Chern-Simons fermion Hall conductance in the dipole theory of the half-filled Landau level, Phys. Rev. B 60, 5636 (1999).

[33] V. Pasquier and F. D. M. Haldane, A dipole interpretation of the $v=1 / 2$ state, Nucl. Phys. B 516, 719 (1998).

[34] F. von Oppen, B. I. Halperin, S. H. Simon, and A. Stern, Half-filled Landau level-Composite fermions and dipoles, in Advances in Solid State Physics 39, edited by B. Kramer (Springer, Berlin, 1999), pp. 203-212.

[35] C. Wang and T. Senthil, Half-filled Landau level, topological insulator surfaces, and three-dimensional quantum spin liquids, Phys. Rev. B 93, 085110 (2016).

[36] G. Murthy and R. Shankar, $v=\frac{1}{2}$ Landau level: Half-empty versus half-full, Phys. Rev. B 93, 085405 (2016). 
[37] D. Gočanin, S. Predin, M. D. Ćirić, V. Radovanović, and M. Milovanović, Microscopic derivation of Dirac composite fermion theory: Aspects of noncommutativity and pairing instabilities, Phys. Rev. B 104, 115150 (2021).

[38] J. D. Jackson, Classical Electrodynamics (Wiley, Hoboken, NJ, 1999).

[39] D. Xiao, J. Shi, and Q. Niu, Berry Phase Correction to Electron Density of States in Solids, Phys. Rev. Lett. 95, 137204 (2005).

[40] P. A. Horváthy, The Non-commutative Landau Problem, Ann. Phys. (NY) 299, 128 (2002).

[41] S. D. Geraedts, J. Wang, E. H. Rezayi, and F. D. M. Haldane, Berry Phase and Model Wave Function in the Half-Filled Landau Level, Phys. Rev. Lett. 121, 147202 y (2018).

[42] N. A. Sinitsyn, Q. Niu, and A. H. MacDonald, Coordinate shift in the semiclassical Boltzmann equation and the anomalous Hall effect, Phys. Rev. B 73, 075318 (2006).

[43] M.-C. Chang and Q. Niu, Berry curvature, orbital moment, and effective quantum theory of electrons in electromagnetic fields, J. Phys.: Condens. Matter 20, 193202 (2008).

[44] G. Ji and J. Shi, Berry phase in the composite Fermi liquid, Phys. Rev. Research 2, 033329 (2020).

[45] J. Callaway, Quantum Theory of the Solid State (Academic Press, New York, 1991).

[46] A. D. Mirlin and P. Wölfle, Composite Fermions in the Fractional Quantum Hall Effect: Transport at Finite Wave Vector, Phys. Rev. Lett. 78, 3717 (1997).

[47] D. X. Nguyen, S. Golkar, M. M. Roberts, and D. T. Son, Particle-hole symmetry and composite fermions in fractional quantum Hall states, Phys. Rev. B 97, 195314 (2018).

[48] D. Kamburov, Y. Liu, M. Shayegan, L. N. Pfeiffer, K. W. West, and K. W. Baldwin, Composite Fermions with Tunable Fermi Contour Anisotropy, Phys. Rev. Lett. 110, 206801 (2013).

[49] D. Kamburov, Y. Liu, M. A. Mueed, M. Shayegan, L. N. Pfeiffer, K. W. West, and K. W. Baldwin, What Determines the Fermi Wave Vector of Composite Fermions?, Phys. Rev. Lett. 113, 196801 (2014).
[50] S. H. Simon, A. Stern, and B. I. Halperin, Composite fermions with orbital magnetization, Phys. Rev. B 54, R11114 (1996).

[51] G. Giuliani and G. Vignale, Quantum Theory of the Electron Liquid (Cambridge University Press, Cambridge, 2005).

[52] Y. Gao, S. A. Yang, and Q. Niu, Geometrical effects in orbital magnetic susceptibility, Phys. Rev. B 91, 214405 (2015).

[53] A. Wixforth, J. Scriba, M. Wassermeier, J. P. Kotthaus, G. Weimann, and W. Schlapp, Surface acoustic waves on GaAs/ $/ \mathrm{Al}_{\mathrm{x}} \mathrm{Ga}_{1-\mathrm{x}} \mathrm{As}$ heterostructures, Phys. Rev. B 40, 7874 (1989).

[54] R. L. Willett, M. A. Paalanen, R. R. Ruel, K. W. West, L. N. Pfeiffer, and D. J. Bishop, Anomalous Sound Propagation at $v=1 / 2$ in a 2D Electron Gas: Observation of a Spontaneously Broken Translational Symmetry?, Phys. Rev. Lett. 65, 112 (1990).

[55] R. L. Willett, R. R. Ruel, M. A. Paalanen, K. W. West, and L. N. Pfeiffer, Enhanced finite-wave-vector conductivity at multiple even-denominator filling factors in two-dimensional electron systems, Phys. Rev. B 47, 7344 (1993).

[56] B. K. Ridley, Space charge waves and the piezo-electric interaction in 2D semiconducting structures, Semicond. Sci. Technol. 3, 542 (1988).

[57] R. L. Willett, K. W. West, and L. N. Pfeiffer, Apparent Inconsistency of Observed Composite Fermion Geometric Resonances and Measured Effective Mass, Phys. Rev. Lett. 75, 2988 (1995).

[58] R. L. Willett, R. R. Ruel, K. W. West, and L. N. Pfeiffer, Experimental Demonstration of a Fermi Surface at One-Half Filling of the Lowest Landau Level, Phys. Rev. Lett. 71, 3846 (1993).

[59] S. H. Simon, Coupling of surface acoustic waves to a twodimensional electron gas, Phys. Rev. B 54, 13878 (1996).

[60] P. Nozières, Theory of Interacting Fermi Systems (AddisonWesley, New York, 1997).

[61] The constant has an oscillatory dependence on $d$. Our own calculation shows the same qualitative behavior as that shown in Ref. [59] but with quantitative differences. It indicates that the constant has a maximal value $3.7 \times 10^{-4}$ at $d=0$ and a second peak $3.1 \times 10^{-4}$ at $q d \sim 2.5$. 